AperTO - Archivio Istituzionale Open Access dell'Università di Torino

\title{
Tiered objects
}

\section{This is the author's manuscript}

Original Citation:

Availability:

This version is available http://hdl.handle.net/2318/1633801

since 2020-01-21T14:25:31Z

Published version:

DOI:10.3233/FI-2016-1449

Terms of use:

Open Access

Anyone can freely access the full text of works made available as "Open Access". Works made available under a Creative Commons license can be used according to the terms and conditions of said license. Use of all other works requires consent of the right holder (author or publisher) if not exempted from copyright protection by the applicable law. 


\title{
Tiered objects
}

\author{
Fabio Alessi \\ Dipartimento di Matematica e Informatica, Università di Udine, \\ via delle Scienze 208, I-33100 Udine, Italy.
}

\section{Felice Cardone}

Dipartimento di Informatica, Università di Torino, corso Svizzera 185, I-10149 Torino, Italy.

\begin{abstract}
We investigate the foundations of reasoning over infinite data structures by means of set-theoretical structures arising in the sheaf-theoretic semantics of higher-order intuitionistic logic. Our approach focuses on a natural notion of tiering involving an operation of restriction of elements to levels forming a complete Heyting algebra. We relate these tiered objects to final coalgebras and initial algebras of a wide class of endofunctors of the category of sets, and study their order and convergence properties. As a sample application, we derive a general proof principle for tiered objects.
\end{abstract}

Keywords: complete Heyting algebras, sheaves, initial algebras, final coalgebras, infinite data structures, approximation lemma

$$
\begin{aligned}
& \text { A Mario e Mariangiola, } \\
& \text { per i loro } 70 \text { anni }
\end{aligned}
$$

\section{Introduction}

Among the many frameworks that have been proposed in order to deal with infinite objects in a computational context, an important place is taken by structures whose elements are cumulatively arranged in layers according to a general notion of rank taking values in the set of extended natural numbers $0,1,2, \ldots, \infty$. Elements have approximated versions at each layer, so that each element can be seen as a limit of the sequence of its approximations, in analogy to what happens with fundamental sequences for limit ordinals. Structures of this kind have appeared in many guises in the literature. Without aiming at an exhaustive list, we mention the rank-ordered sets introduced in [1], motivated by the semantics of recursive types and later extended in several ways, see [2, 3]. Closely related structures 
are the complete projection spaces, [4], that arose independently from work on the projective model of process algebra [5, 6]. The families of equivalence relations studied in [7] and (complete) ultrametric spaces [8, $\S \S 8.1-.2]$ (see also [9]) are further ways of formalizing the same basic intuitions, together with the partial metric spaces of $[10,11]$, where the stratification of elements is obtained by allowing elements that have non-zero distance from themselves.

Central to all these structures is a notion of tiering. The starting point of the present paper is the observation that, in many interesting cases, tiered objects can be regarded as a special case of (pre)sheaves over a complete Heyting algebra as formulated by Fourman and Scott [12] in their work on the sheaf-theoretic semantics of intuitionistic higher-order logic. This provides an elegant framework for discussing general properties of infinite objects as made up of their initial segments of finite length, which is the theme underlying all the structures mentioned above. While this theme as an instance of the passage from local to global which is typical of sheaves [13], our simplified setting also supports in a straightforward manner many of the calculations involving sequences of approximations to infinite objects that arise in related formalisms.

Important examples of tiered objects come from final coalgebras. For example, the set of finite and infinite words over a non-empty alphabet $\Sigma$, namely $\Sigma^{\infty}={ }_{\text {def }} \Sigma^{*} \cup \Sigma^{\omega}$ is the final coalgebra of the polynomial endofunctor of Set which assigns to a set $X$ the set $\{\varepsilon\} \cup(\Sigma \times X)$. It is known that such coalgebras can be regarded both as complete ultrametric spaces [14] and as complete partial orders [15]. However, by making explicit their tiering structure, inductive arguments can be carried out in the place of coinductive proofs that exploit bisimulations in order to prove equality of lazy data structures [16]. In this respect, our work bears on research described, for example, in [10] where the role of tiering is played by sequences of equivalence relations indexed over a well-founded set, allowing recursive definitions over non-inductive types.

The outline of the paper is as follows. After defining tiered objects and proving their basic properties in Section 2, in Section 3 we display examples demonstrating the extent to which tiered objects subsume common approaches to spaces of infinite structures. These examples include finite and infinite words, recursively defined functions, and final coalgebras of $\omega$-continuous set functors. In Section 4, we discuss completeness of tiered objects and show the equivalence of order-theoretic completeness with Cauchy completeness, where Cauchy sequences are defined by exploiting a natural ultrametric or, equivalently, the tiering structure. The latter leads very naturally to a notion of projective completion for tiered objects, which is studied in Section 5. In Section 6 we investigate the relation between tiered objects and certain pairs of direct and inverse sequences in the category of sets, named tierable pairs. In the last Section we investigate sufficient conditions on endofunctors of Set so that their final coalgebras are projective completions of initial algebras, applying general results developed in Section 6 and carrying over into the tiered framework results of Adámek [15]. Finally, we set up a proof principle for tiered objects that seems to encapsulate the form of reasoning that lies at the basis of some approaches to proving properties of infinite objects, in particular the coinductive proof system for subtyping recursive types described in [17], or the guarded induction principle of [18].

\section{Tiered objects}

In order to define tiered objects we first notice the existence of a familiar algebraic structure on the extended natural numbers (the tiers) $0,1,2, \ldots, \infty$.

Definition 2.1. A complete Heyting algebra (cHa) is a complete lattice $H$ satisfying the distributive 
law:

$$
a \wedge \bigvee X=\bigvee_{x \in X}(a \wedge x)
$$

for any $X \subseteq H$.

Proposition 2.2. $\Omega=_{\text {def }}\{0<1<\cdots<\infty\}$ is a complete Heyting algebra.

The cHa $\Omega$ is not a Boolean algebra. Its logical properties are studied in [19]. In the sequel, by $\Omega$ we shall always mean the cHa of tiers. Tiered objects, to be introduced presently, are in fact presheaves over $\Omega$ presented, as in [12], by exploiting Heyting-valued restriction and extent operations (see also $[20,21])$. We shall not commit to any notion of morphism between tiered objects: this explains the choice of a new name for these structures. Intuitively, a tiered object consists of a set $A$ whose elements $a$ have approximated versions $a\lceil p$ at every stage $p \in \Omega$. Every element $a$ has an extent $\mathbf{E} a \in \Omega$, which is the stage at which $a$ becomes fully defined, so that $a=a\lceil p$ for every $p \geq \mathbf{E} a$.

Definition 2.3. (Tiered objects)

A structure

$$
\mathbf{A}=\langle A, \cdot \uparrow: A \times \Omega \rightarrow A, \mathbf{E}: A \rightarrow \Omega\rangle
$$

is a tiered object if the following equations hold for all $p, q \in \Omega, a \in A$ :

$$
\begin{aligned}
a \uparrow \mathbf{E} a & =a \\
(a \uparrow p) \uparrow q & =a \uparrow(p \wedge q) \\
\mathbf{E}(a \uparrow p) & =\mathbf{E} a \wedge p .
\end{aligned}
$$

The operations $\uparrow$ and $\mathbf{E}$ are called restriction and extent respectively.

Proposition 2.4. For any $a \in A, \mathbf{E} a$ is the least $p \in \Omega$ such that $a \uparrow p=a$.

Proof:

By equation (1), $a=a \uparrow \mathbf{E} a$. If $a=a \uparrow p$, then $\mathbf{E} a=\mathbf{E}(a \uparrow p)=\mathbf{E} a \wedge p$ by equation (3), therefore $\mathbf{E} a \leq p$.

We say that two tiered object $\mathbf{A}$ and $\mathbf{B}$ are isomorphic if there exists a bijection $f: A \rightarrow B$ that respects restriction and extent: for any $a \in A, p \in \Omega$,

- $f\left(a \uparrow_{\mathbf{A}} p\right)=f(a) \uparrow_{\mathbf{B}} p$;

- $\mathbf{E}_{\mathbf{B}} f(a)=\mathbf{E}_{\mathbf{A}} a$.

Remark 2.5. The axioms for tiered objects are almost the same as those of rank-ordered sets [1] (and also those of projection spaces [4]). Structures of both kinds satisfy equations (1) and (2). What is missing from rank-ordered sets is a notion of extent satisfying Equation 3). As an example of a rankordered set that is not a tiered object, consider "partial streams," namely functions $\sigma: \omega \rightarrow A \cup\{*\}$, where $\sigma(i)=*$ means intuitively that position $i$ in stream $\sigma$ is empty. Call $\sigma_{*}$ the partial stream $\sigma(i)=*$, for any $i$. For a partial stream $\sigma$, define

$$
\mathcal{I}(\sigma)=\{i \mid \sigma(i) \neq *\}
$$


Then define

$$
\mathbf{E} \sigma= \begin{cases}0 & \text { if } \sigma=\sigma_{*} \\ \max (\mathcal{I}(\sigma))+1 & \text { if } \mathcal{I}(\sigma) \text { is non-empty and bounded } \\ \infty & \text { otherwise }\end{cases}
$$

Restriction is defined as

$$
\left(\sigma\lceil p)(i)= \begin{cases}\sigma(i) & \text { if } i<p \\ * & \text { otherwise }\end{cases}\right.
$$

It is easy to check that (1) and (2) hold for partial streams, but (3) fails.

\subsection{Tiered objects as partial orders}

It is precisely the interaction of extent and restriction in tiered objects that affords a straightforward way of defining a partial order relation on their elements.

Definition 2.6. For $\mathbf{A}$ a tiered object and $a, b \in A$, define

$$
a \sqsubseteq b \Leftrightarrow a=b\lceil\mathbf{E} a .
$$

Define $a, b \in A$ to be compatible, written $a \gamma b$, if $a \nmid \mathbf{E} b=b \uparrow \mathbf{E} a . B \subseteq A$ is compatible if its elements are pairwise compatible.

This use of extent and restriction in defining a partial order on the elements of a tiered object is similar, and to some extent dual, to the use of distance in defining a partial order on partial metric spaces as described in $[11, \S 3]$. The precise relations between these two settings deserves further investigation. Intuitively, $a$ and $b$ are compatible if they agree up to the least among $\mathbf{E} a$ and $\mathbf{E} b$, because it is easy to see that

$$
a \searrow b \quad \text { iff } \quad a \uparrow(\mathbf{E} a \wedge \mathbf{E} b)=b \uparrow(\mathbf{E} a \wedge \mathbf{E} b) .
$$

Clearly, $a \varnothing b$ if and only if $a$ and $b$ are comparable under $\sqsubseteq$, see Proposition 2.7(6) below. Compatibility is however useful to ease comparison with the axiomatization of sheaves in [12, 20].

We collect now the basic order properties of tiered objects (see also [12]): the straightforward proofs, demonstrating in particular how natural is the axiomatization based on extent and restriction, are omitted. Recall that a chain is a set of elements that are pairwise comparable under $\sqsubseteq$; a set $X$ is directed if any two elements of $X$ have an upper bound in $X$ w.r.t. $\sqsubseteq$.

Proposition 2.7. Assume that $\mathbf{A}$ is a tiered object. Then, for all $a, b, c \in A$ :

1. $a \sqsubseteq b \Longrightarrow \mathbf{E} a \leq \mathbf{E} b$.

2. $a \sqsubseteq b, b \sqsubseteq c \Longrightarrow a \sqsubseteq c$.

3. $a \sqsubseteq b, b \sqsubseteq a \Longrightarrow a=b$.

4. For any $p, q \in \Omega, p \leq q \Longrightarrow a \uparrow p \sqsubseteq a \uparrow q$.

5. For any $p \in \Omega, a \sqsubseteq b \Longrightarrow a \uparrow p \sqsubseteq b \uparrow p$. 
6. If $X \subseteq A$ is directed, then it is a countable chain. In particular, every compatible subset of a tiered object is a countable chain.

7. $a \uparrow p=a \uparrow(p+1) \Longrightarrow \mathbf{E} a \leq p$.

8. $a=b\lceil p \Longrightarrow a \sqsubseteq b$.

9. $a \sqsubseteq b, \mathbf{E} a=\mathbf{E} b \Longrightarrow a=b$.

10. If $B$ is a infinite chain, then $\bigvee_{b \in B} \mathbf{E} b=\infty$.

11. $a=b\lceil p, \mathbf{E} a \neq p \Longrightarrow a=b$.

12. $p \geq \mathbf{E} a \Longrightarrow a=a \uparrow p$.

As in the Heyting-valued approach to (pre)sheaves, completeness and separation properties play an important role. For defining them we just need to use countable chains, since by Proposition 2.7(6), in a tiered object compatible or directed subsets turn out to be chains.

Definition 2.8. [Separation and completeness] Let $\mathbf{A}=\langle A, \cdot \uparrow \cdot: A \times \Omega \rightarrow A, \mathbf{E}: A \rightarrow \Omega\rangle$ be a tiered object, and let $B \subseteq A$.

1. B is bounded if it has some $\sqsubseteq$-upper bound.

2. A join for $B \subseteq A$ is a $\sqsubseteq$-minimal upper bound for $B$.

3. A is separated if every subset of $A$ that has a join has a unique join.

4. A is complete if every compatible $B$ has a unique join.

5. A is pointed if there exists an element $\perp \in A$ such that $\perp=a\lceil 0$, for all $a \in A$.

Observe that any complete tiered object $\mathbf{A}$ is separated, by definition. It is also pointed, because the empty set $\emptyset$ is compatible and therefore has a unique join $\perp$; any $a \in A$ is such that $a \uparrow 0$ is a minimal upper bound of $\emptyset$, hence $a\lceil 0=\perp$. The following Proposition characterizes joins. Points (2) and (3) reproduce Lemmas 4.5(iii) and (iv) from [12]. They entail, in particular, that separated tiered objects have least upper bounds of compatible subsets.

Proposition 2.9. Let $\mathbf{A}=\langle A, \cdot \uparrow \cdot: A \times \Omega \rightarrow A, \mathbf{E}: A \rightarrow \Omega\rangle$ be a tiered object, and $a \in A$ :

1. Let $B \subseteq A$ be a bounded infinite chain. Then any upper bound of $B$ is a join.

2. $a$ is a join for a bounded chain $B \subseteq A$ if and only if $\mathbf{E} a=\bigvee_{b \in B} \mathbf{E} b$ and $b \sqsubseteq a$ for all $b \in B$.

3. $a \uparrow \bigvee_{i \in I} p_{i}$ is a join for $\left\{a\left\lceil p_{i} \mid i \in I\right\}\right.$ for all $a \in A$ and $\left\{p_{i} \mid i \in I\right\} \subseteq \Omega$.

\section{Proof:}

(1) Let $B$ be bounded and $c$ be any upper bound for $B$ (hence for any $b \in B, b \sqsubseteq c$ ). We prove that $c$ is minimal. Suppose that $a$ is another upper bound for $B$, and let $a \sqsubseteq c$. By Proposition 2.7(10) and 2.7(1), $\mathbf{E} a=\mathbf{E} c=\infty$. Then we apply 2.7(9) and derive $a=c$, hence $c$ is minimal.

(2) $(\Rightarrow)$ Let $B$ be bounded and $a$ be a join for $B$. If $B$ is finite, then $a=\max B$, hence $a$ satisfies 
the thesis. Otherwise $\bigvee_{b \in B} \mathbf{E} b=\infty$ by Proposition 2.7(10), hence by Proposition 2.7(1) we have $\mathbf{E} a=\infty$, since $b \sqsubseteq a$ for any $b \in B$, and the thesis follows.

$(\Leftarrow)$ If $B$ is finite, then $\mathbf{E} a=\bigvee_{b \in B} \mathbf{E} b$ implies that there is $b^{\prime} \in B$ such that $\mathbf{E} a=\mathbf{E} b^{\prime}$. Since $b^{\prime} \sqsubseteq a$, $a=b^{\prime}$ by Proposition 2.7(9), hence $a \in B$ is the least upper bound of $B$ (hence a join), being the greatest element in $B$. If $B$ is infinite, then the thesis follows immediately by the previous point (1), since by hypothesis $a$ is an upper bound of $B$.

(3) Let $p^{\prime}=\bigvee_{i \in I} p_{i}$. We have, for any $i \in I, a\left\lceil p_{i} \sqsubseteq a\left\lceil p^{\prime}\right.\right.$ by Proposition 2.7(4). If $p^{\prime} \in \omega$, the thesis is immediate, since $p^{\prime}=p_{j}$ for some $j \in I$, and $a \uparrow p_{j}$, as the greatest element of the chain $\left\{a\left\lceil p_{i} \mid i \in I\right\}\right.$, is the least upper bound (hence a join). If $p^{\prime}=\infty$, then observe $a \uparrow p^{\prime}=a$. Let $c \sqsubseteq a$ be another upper bound for $\left\{a \uparrow p_{i} \mid i \in I\right\}$. Then we have, for any $i \in I, \mathbf{E}\left(a \uparrow p_{i}\right) \leq \mathbf{E} c$, that is $\mathbf{E} a \wedge p_{i} \leq \mathbf{E} c$. Since the set $\left\{p_{i} \mid i \in I\right\}$ is unbounded, this implies $\mathbf{E} a \leq \mathbf{E} c$. On the other hand, $c \sqsubseteq a$ implies, by Proposition 2.7(1), $\mathbf{E} c \leq \mathbf{E} a$, hence $\mathbf{E} c=\mathbf{E} a$. We conclude $a=c$ by Proposition 2.7(9). Therefore $a$ is minimal and the proof is complete.

Points (2), (3), and (4) of the next lemma show that extent and restriction are Scott continuous operations. The last point shows that approximation is cumulative.

Lemma 2.10. Let $\mathbf{A}$ be a separated tiered object, and let $B \subseteq A$ be a bounded chain. Then

1. $B$ has a least upper bound, denoted $\bigsqcup B$.

2. $\mathbf{E}(\bigsqcup B)=\bigvee_{b \in B} \mathbf{E} b$.

3. For any $p \in \Omega,(\bigsqcup B) \uparrow p=\bigsqcup_{b \in B}(b\lceil p)$.

4. For any $a \in A$ and $\left\{p_{i} \mid i \in I\right\} \subseteq \Omega, a \uparrow\left(\bigvee_{i \in I} p_{i}\right)=\bigsqcup_{i \in I}\left(a\left\lceil p_{i}\right)\right.$.

5. For any $a \in A, a=\bigsqcup_{n \in \omega}(a\lceil n)$.

\section{Proof:}

(1) If $B$ is finite the thesis is trivial, since the greater element of $B$ is its least upper bound. If $B$ is infinite, we have that any upper bound of $B$ is a join for $B$, by Proposition 2.9(1). On the other hand by definition of separated tiered object, $B$ has a unique join, hence $B$ has a unique minimal upper bound, which is of course the least one.

(2) $b \sqsubseteq \bigsqcup B$ implies $\mathbf{E} b \leq \mathbf{E}(\bigsqcup B)$ by Proposition 2.7(1), hence $\bigvee_{b \in B} \mathbf{E} b \leq \mathbf{E}(\bigsqcup B)$. Conversely, if $B$ is finite, we have $\mathbf{E}(\bigsqcup B) \leq \bigvee_{b \in B} \mathbf{E} b$, since $\bigsqcup B \in B$. If $B$ is infinite, then $\bigvee_{b \in B} \mathbf{E} b=\infty$ by Proposition 2.7(10), hence $\mathbf{E}(\bigsqcup B) \leq \bigvee_{b \in B} \mathbf{E} b$.

(3) First note that the chain $\{b|p| b \in B\}$ is bounded, hence by the previous point (1) it has a unique join. We have $\bigsqcup_{b \in B}(b \uparrow p) \sqsubseteq(\bigsqcup B) \uparrow p$, since by Proposition 2.7(5), for any $b \in B$, 
$b\lceil p \sqsubseteq(\bigsqcup B) \uparrow p$. Moreover

$$
\begin{aligned}
\mathbf{E}\left(\bigsqcup_{b \in B}(b \uparrow p)\right) & =\bigvee_{b \in B} \mathbf{E}(b\lceil p) \\
& =\bigvee_{b \in B}((\mathbf{E} b) \wedge p) \\
& =\left(\bigvee_{b \in B} \mathbf{E} b\right) \wedge p \\
& =\mathbf{E}(\bigsqcup B) \wedge p \\
& =\mathbf{E}((\bigsqcup B) \uparrow p)
\end{aligned}
$$

hence we conclude using Proposition 2.7(9).

(4) This point is just Proposition 2.9(3), rephrased with the notation for least upper bounds.

(5)

$$
\begin{aligned}
\bigsqcup_{n \in \omega}(a \uparrow n) & =a \uparrow\left(\bigvee_{n \in \omega} n\right) \quad \text { by point (3) } \\
& =a \uparrow \infty \\
& =a
\end{aligned}
$$

Corollary 2.11. Let $\mathbf{A}$ be a complete tiered object. Then any chain has a least upper bound. Moreover A is a cpo.

\section{Proof:}

The first statement is an immediate consequence of Lemma 2.10(1) and definition of complete tiered object. The second statement follows from the first, since by Proposition 2.7(6) any directed set is a chain.

The following immediate consequence of Lemma 2.10(5) has an independent interest: it can be seen as a proof principle for complete tiered objects akin to the Approximation Lemma used in functional programming $[22,23]$.

\section{Lemma 2.12. (The approximation lemma)}

If $\mathbf{A}$ is separated,

$$
(\forall n \in \omega . \quad a\lceil n=b\lceil n) \Longrightarrow a=b .
$$

\section{Examples}

\subsection{Finite and infinite words}

As a preview of the interpretation of tiering just introduced, we sketch how it arises in the case of the set $\Sigma^{\infty}$ of finite and infinite words. 
Given any non-empty set $\Sigma$ (of "letters"), a finite or infinite word $u$ over $\Sigma$ is a mapping $u$ : $\gamma \rightarrow \Sigma$ for some (von Neumann) ordinal $\gamma \leq \omega$. The empty word $\varepsilon$ is the empty map $\emptyset \rightarrow \Sigma$. Therefore $\Sigma^{\infty}$ can be identified with $\bigcup_{n \in \omega} \Sigma^{n} \cup \Sigma^{\omega}$. Every element $u \in \Sigma^{\infty}$ can be given extent $\mathbf{E}(u)=_{\text {def }} \operatorname{dom}(u) \leq \omega$. Besides, for any $\gamma$, consider the restriction $u\left\lceil\gamma\right.$ defined as $u_{\mid \operatorname{dom}(u) \cap \gamma}$, and observe that for any $\gamma, \delta \leq \omega$ :

$$
\begin{aligned}
(u\lceil\gamma) \uparrow \delta & =u\lceil\gamma \cap \delta & & \text { (by properties of restriction) } \\
& =u\lceil\min (\gamma, \delta) & & \text { (by properties of von Neumann ordinals) } \\
& =(u\lceil\delta) \uparrow \gamma . & &
\end{aligned}
$$

The equations defining $\Sigma^{\infty}$ as a tiered object are satisfied

$$
\begin{aligned}
& u \uparrow \mathbf{E}(u)=u, \\
& \mathbf{E}(u \uparrow \gamma)=\mathbf{E}(u) \cap \gamma
\end{aligned}
$$

and follow immediately from the definition of restriction and extent. For words $u, v \in \Sigma^{\infty}$ we have

$$
u \curlyvee v \quad \text { iff } \quad u \uparrow \operatorname{dom}(v)=v \uparrow \operatorname{dom}(u) .
$$

If $W \subseteq \Sigma^{\infty}$ is compatible, let the join of $W$ be the function

$$
(\bigsqcup W)(\alpha)=u(\alpha)
$$

for any $u$ such that $\alpha \in \mathbf{E}(u)$, where, by definition,

$$
\mathbf{E}(\bigsqcup W)=\bigcup_{w \in W} \mathbf{E}(w) .
$$

Compatibility of $W$ guarantees that this is indeed a function. In fact, if $\alpha \in \mathbf{E}(u) \cap \mathbf{E}(v)$ :

$$
\begin{aligned}
u(\alpha) & =(u \uparrow \mathbf{E}(v)) & & (\text { as } \alpha \in \mathbf{E}(u) \cap \mathbf{E}(v)) \\
& =(v \uparrow \mathbf{E}(u)) & & (\text { as } u \gamma v) \\
& =v(\alpha) . & &
\end{aligned}
$$

Observe that $u \sqsubseteq v$ precisely when $u \subseteq v$ as functions, so $\bigsqcup W$ is the least upper bound of $W$ w.r.t. $\sqsubseteq$. Therefore $\Sigma^{\infty}$ is a complete tiered object.

\subsection{Recursion}

While tiering is especially useful in situations involving structures of infinite depth, it also shows up in a context which is intimately related to induction over the natural numbers, namely the justification of the iterative definition of functions contained in the classical work of Dedekind [24]. Following [25], we briefly summarize this landmark result by highlighting its relations to tiering. Given a structure $\mathcal{A}=\langle A, a \in A, f: A \rightarrow A\rangle$, in order to show that there exists a unique function $\omega \rightarrow A$ such that:

$$
\begin{aligned}
h(0) & =a \\
h(n+1) & =f(h(n))
\end{aligned}
$$

we build a set of approximations of $h$ having as domains segments $H \subseteq \omega$ such that 
- $0 \in H$;

- if $n+1 \in H$, then $n \in H$.

Clearly, the collection of segments of $\omega$ ordered by inclusion is isomorphic to $\Omega$. A partial function is any single-valued relation $\varphi \subseteq \omega \times A$ whose domain is a segment and that satisfies

$$
\begin{aligned}
\varphi(0) & =a \\
\varphi(n+1) & =f(\varphi(x))
\end{aligned}
$$

whenever $n+1 \in \operatorname{dom}(\varphi)$. The construction of $h$ proceeds by proving that

1. for every $n \in \omega$ there exists a partial function $\varphi$ such that $n \in \operatorname{dom}(\varphi)$;

2. if $n \in \operatorname{dom}\left(\varphi_{1}\right) \cap \operatorname{dom}\left(\varphi_{2}\right)$, then $\varphi_{1}(n)=\varphi_{2}(n)$.

Finally, let

$$
h(n)=_{\operatorname{def}} \varphi(n) \quad \text { where } n \in \operatorname{dom}(\varphi) .
$$

It is easy to see that, by defining $\mathbf{E} \varphi={ }_{\operatorname{def}} \operatorname{dom}(\varphi)$ and taking restriction as restriction of a function to a segment, the collection of partial functions is a tiered object. Observe that compatibility of partial functions defined by

$$
\varphi_{1} \varnothing \varphi_{2} \Leftrightarrow\left(\forall n \in \operatorname{dom}\left(\varphi_{1}\right) \cap \operatorname{dom}\left(\varphi_{2}\right) \Rightarrow \varphi_{1}(n)=\varphi_{2}(n)\right)
$$

coincides with their compatibility as elements of a tiered object. The construction of $h$ exploits precisely the completeness of the set of partial functions as a tiered object.

\subsection{Final coalgebras as tiered objects}

There is a close connection between final coalgebras of $\omega$-continuous endofunctors of Set and complete tiered objects. Assume that $F:$ Set $\longrightarrow$ Set is an $\omega$-continuous functor and build the sequence:

$$
T_{0} \leftarrow_{j_{0}} T_{1} \leftarrow_{j_{1}} T_{2} \leftarrow_{j_{2}} \cdots \underset{j_{n-1}}{\leftarrow_{n}} T_{n} \leftarrow_{j_{n}} T_{n+1} \underset{j_{n+2}}{\leftarrow_{n}} \cdots
$$

where:

$$
\begin{aligned}
T_{0} & =\mathbf{1}, \text { where } \mathbf{1} \text { is any singleton } \\
T_{n+1} & =F\left(T_{n}\right)
\end{aligned}
$$

and the pairs $j_{n}$, for $n>0$, are defined inductively by

$$
j_{n}=F\left(j_{n-1}\right): F\left(T_{n}\right) \rightarrow F\left(T_{n-1}\right) .
$$

Observe that a cone for diagram (6) with components:

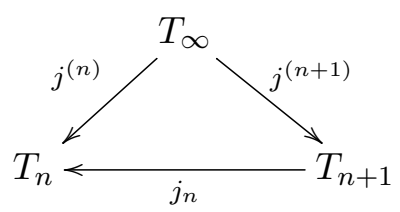


amounts to a presheaf $T: \Omega^{\mathrm{op}} \longrightarrow$ Set where $T(p)=T_{p}$ and, for $p<q \in \omega$, the mapping $T(q) \longrightarrow T(p)$ is the composition $j_{q, p}={ }_{\text {def }} j_{p} \circ \cdots \circ j_{q-1}$. As in [12, 4.14(iii)], a crible $K$ of an element $p$ of $\Omega$ is a set $K \subseteq \Omega$ of elements $q \leq p$ such that $r \leq q \in K$ implies $r \in K$. A crible $K$ of $p$ covers $p$ if $p=\bigvee K$. In general, if $K$ is a covering crible of $p \in \Omega$ there is a cone

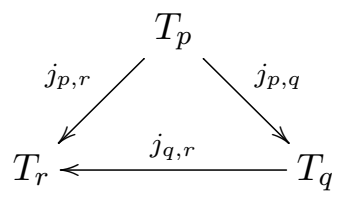

with vertex $T(p)=T_{p}$, for any $p, q \in K: T$ is a sheaf exactly when, for every $p$, the universal mapping towards the limit

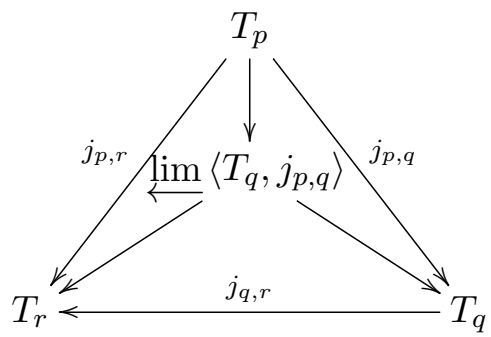

is a bijection. In the case of $\Omega$, this condition is non-trivial only when $K=\omega$ and $p=\infty$, therefore we can conclude

Proposition 3.1. For an $\omega$-continuous $F$ : Set $\longrightarrow$ Set, the presheaf $T: \Omega^{\mathrm{op}} \longrightarrow$ Set associated with the limiting cone

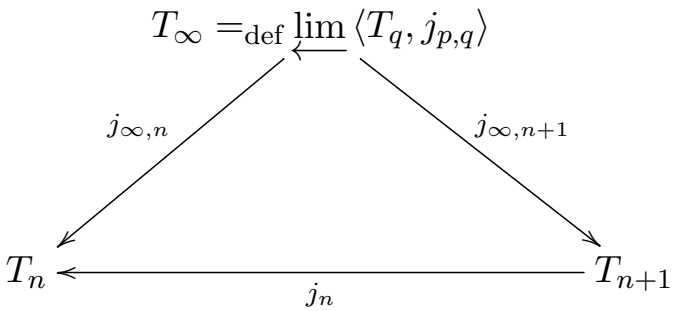

is a sheaf.

The construction of a complete tiered object $\mathbf{T}=\langle A, \cdot \uparrow \cdot A \times \Omega \rightarrow A, \mathbf{E}: A \rightarrow \Omega\rangle$ out of the sheaf $T$ can then be carried out as in $[12$, loc. cit.]:

- $A={ }_{\text {def }} \coprod_{p \in \Omega} T_{p}$;

- for $a=\langle p, t\rangle \in A, \mathbf{E}(a)=p$;

- for $a=\langle p, t\rangle \in A$ and $q \in \Omega: a\left\lceil q=\left\langle p \wedge q, j_{p, p \wedge q}(t)\right\rangle\right.$.

Thus, the complete tiered object $\mathbf{T}$ summarizes the construction of the final $F$-coalgebra, which is carved out of $\mathbf{T}$ as the subset of maximal elements (i.e., those of extent $\infty$ ). Observe that $\mathbf{T}$ is, by Corollary 2.11, a complete partial order (cpo, [8]); it is also easily seen that such a cpo is algebraic, the compact elements being those of finite extent. This suggests that our association of a complete tiered object to the final coalgebra of an $\omega$-continuous endofunctor of Set can be exploited in defining computational models for final coalgebras, in analogy to the computational models of ultrametric spaces described in [26]. However, we shall not pursue this matter any further in the present paper. 


\section{Cauchy completeness}

Let $\mathbf{A}=\langle A, \uparrow, \mathbf{E}\rangle$ be a tiered object. We introduce a notion of (discrete) pseudometric

$$
d: A \times A \rightarrow\{0\} \cup\left\{2^{-n} \mid n \in \omega\right\}
$$

by setting $d(x, y)=2^{-\delta(x, y)}$, where $\delta: A \times A \rightarrow \Omega$ is defined by

$$
\delta(x, y)=\min \{p \mid x \uparrow p \neq y\lceil p\}
$$

$(\delta(x, y)=\infty$ if the set in the right-hand side is empty). In the separated case $d$ is an ultrametric, but in the general case the first axiom of metric fails: consider for instance $\{n \mid n \in \omega\} \cup\{a, b\}$, with $\mathbf{E} n=n, \mathbf{E} a=\mathbf{E} b=\infty$, and for any $m \in \Omega, n\lceil m=\min \{n, m\}, a\lceil m=b\lceil m=m$. Then $d(a, b)=0$, since $\delta(a, b)=\infty$, nevertheless $a \neq b$. A Cauchy sequence in the pseudo-ultrametric space $\langle A, d\rangle$ is a sequence $\left\langle a^{(n)}\right\rangle_{n \in \omega}$ such that

$$
\forall \varepsilon>0 . \exists j(\varepsilon) \in \omega \cdot \forall k \in \omega \cdot d\left(a^{(j(\varepsilon))}, a^{(j(\varepsilon)+k)}\right)<\varepsilon
$$

which is equivalent to

$$
\forall i \in \omega \cdot \exists j(i) \in \omega \cdot \forall k \in \omega \cdot d\left(a^{(j(i))}, a^{(j(i)+k)}\right)<2^{-i}
$$

and also (see for example [27]) to

$$
\forall i \in \omega \cdot \exists j(i) \in \omega \cdot \forall k \in \omega \cdot\left(a^{(j(i))} \uparrow i=a^{(j(i)+k)} \uparrow i\right) .
$$

Convergence of a Cauchy sequence $\left\langle a^{(i)}\right\rangle_{i \in \omega}$ to a limit $a$ is defined by the condition:

$$
\forall i \in \omega \cdot \exists j(i) \in \omega \cdot \forall k \in \omega \cdot\left(a \left\lceili=a^{(j(i)+k)}\lceil i) .\right.\right.
$$

Definition 4.1. A tiered object $\mathbf{A}$ is Cauchy complete if for any Cauchy sequence there exists a unique limit.

We introduce an equivalence relation on Cauchy sequences: let $s=\left\langle a^{(i)}\right\rangle_{i \in \omega}, s^{\prime}=\left\langle b^{(i)}\right\rangle_{i \in \omega}$

$$
s \simeq s^{\prime} \Leftrightarrow \forall i \in \omega \cdot \exists j(i) \in \omega \cdot \forall k, p \in \omega \cdot\left(a^{(j(i)+k)}\left\lceil i=b^{(j(i)+p)} \uparrow i\right) .\right.
$$

We have the following easy lemma.

Lemma 4.2. Given two sequence $s, s^{\prime}$ in a tiered object $\mathbf{A}$, if $s \simeq s^{\prime}$, then $s$ has limit $a$ if and only $a$ is a limit for $s^{\prime}$.

Actually $s \simeq s^{\prime}$ if and only if the distance $d\left(a^{(i)}, b^{(i)}\right) \rightarrow 0$ when $i \rightarrow \infty$. The following simple notion of projective sequence (used, among others, by $[1,27]$ ) will turn out to be equivalent to that of Cauchy sequence, simplifying later proofs: 
Definition 4.3. (Projective completeness)

A sequence $s=\left\langle a^{(i)}\right\rangle_{i \in \omega}$ of elements from a tiered object $\mathbf{A}$ is a projective sequence if

$$
a^{(i)}=a^{(i+1)}\lceil i \text { for all } i \in \omega .
$$

We say that $s$ is $\mathbf{E}$-unbounded if $\bigvee_{i \in \omega} \mathbf{E} a^{(i)}=\infty$

A projective limit for $s$ is any $a \in A$ such that, for all $n \in \omega$ :

$$
a^{(n)}=a \uparrow n .
$$

A is projectively complete if, for every projective sequence $\left\langle a^{(i)}\right\rangle_{i \in \omega}$, there is a unique projective limit, denoted by $a^{(\infty)}$.

Proposition 4.4. Let $s=\left\langle a^{(i)}\right\rangle_{i \in \omega}$ be a projective sequence. Then

1. $\forall i, k \in \omega \cdot a^{(i)}=a^{(i+k)} \uparrow i$. In particular, $s$ is a Cauchy sequence.

2. $s$ is a $\sqsubseteq$-chain $a^{(0)} \sqsubseteq a^{(1)} \sqsubseteq \cdots \sqsubseteq a^{(n)} \sqsubseteq a^{(n+1)} \cdots$.

3. $\forall i \in \omega . \mathbf{E} a^{(i)} \leq i$.

4. $\mathbf{E} a^{(i)}<i \Longrightarrow \forall j \geq i . a^{(j)}=a^{(i)}$.

5. If $s$ is E-unbounded, then $\forall i \in \omega$. $\mathbf{E} a^{(i)}=i$.

6. $a$ is a limit for $s$ if and only if $a$ is a projective limit for $s$.

\section{Proof:}

(1) easy by induction.

(2) by Proposition 2.7(8).

(3) $a^{(i)}=a^{(i+1)} \uparrow i$ implies

$$
\begin{aligned}
\mathbf{E} a^{(i)} & =\mathbf{E}\left(a^{(i+1)} \uparrow i\right) \\
& =\mathbf{E} a^{(i+1)} \wedge i \\
& \leq i .
\end{aligned}
$$

(4) By Proposition 2.7(11), we have immediately $a^{(i+1)}=a^{(i)}$. The thesis follows by induction.

(5) By (3), $\mathbf{E} a^{(i)} \leq i$, for all $i \in \omega$. On the other hand, if $s$ is $\mathbf{E}$-unbounded, the case $\mathbf{E} a^{(i)}<i$ never occurs, otherwise (4) would lead to a contradiction. Therefore the thesis follows.

(6) $(\Leftarrow)$ Let $a$ be a projective limit of $s$, hence $a\left\lceil i=a^{(i)}\right.$. Define $j(i)=i$ in equation (8). We have, by point (1), $a^{(i+k)} \uparrow i=a^{(i)}$, for any $k$, hence we have $a \uparrow i=a^{(i+k)} \uparrow i$ : this proves that $a$ is a limit for $s$.

$(\Rightarrow)$ Suppose $a$ is a limit for $s$. Then for any $i$ there exists $j(i)$ such that for any $k, a^{(j(i)+k)}\lceil i=a\lceil i$. On the other hand $a^{(j(i)+k)} \uparrow i=a^{(i)}$, by $(1)$, since $i \leq j(i)+k$, hence we conclude $a\left\lceil i=a^{(i)}\right.$.

We will prove that the three notions of completeness are equivalent: a tiered object $\mathbf{A}$ is complete if and only if it is Cauchy complete, if and only if it is projectively complete.

We start with a lemma. 
Lemma 4.5. In a tiered object $\mathbf{A}$, for any Cauchy sequence $s=\left\langle a^{(i)}\right\rangle_{i \in \omega}$ there is a unique projective sequence $\pi(s)=\left\langle c^{(i)}\right\rangle_{i \in \omega}$ such that $s \simeq \pi(s)$.

\section{Proof:}

Given a Cauchy sequence $s=\left\langle a^{(i)}\right\rangle_{i \in \omega}$ we define $\pi(s)=\left\langle c^{(i)}\right\rangle_{i \in \omega}$ by:

$$
\begin{gathered}
c^{(i)}=\operatorname{def} a^{(\nu(i))}\lceil i \text { for the least } \nu(i) \text { such that } i \leq \nu(i) \\
\text { and } \forall k \in \omega\left(a ^ { ( \nu ( i ) ) } \left\lceili=a^{(\nu(i)+k)}\lceil i)\right.\right.
\end{gathered}
$$

Observe that

$$
n \leq m \Longrightarrow \nu(n) \leq \nu(m) .
$$

In fact:

(a) $a^{(\nu(m))}\left\lceil n=a^{(\nu(m)+k)} \uparrow n\right.$ for all $k \in \omega$, (use restriction to $n$ and $m$ as from Definition 2.3(2));

(b) $n \leq \nu(m)$ (since by definition $m \leq \nu(m)$ );

then conclude using the minimality of $\nu(n)$ with respect to (a) and (b).

$\pi(s)$ is projective, since

$$
\begin{array}{rlr}
c^{(i+1)} \uparrow i & =a^{(\nu(i+1))} \uparrow i & \\
& =a^{(\nu(i))} \uparrow i & \text { by definition of } \nu(i) \\
& =c^{(i)} . &
\end{array}
$$

We have $s \simeq \pi(s)$. In fact, given $i \in \omega$, for any $k \in \omega$ we have $a^{(\nu(i)+k)} \uparrow i=c^{(i)}$, by definition of $\nu(i)$. On the other hand, for any $p \in \omega$,

$$
\begin{array}{rlr}
c^{(\nu(i)+p)} \uparrow i & =\left(a^{(\nu(\nu(i)+p))} \uparrow(\nu(i)+p)\right) \uparrow i & \\
& =a^{(\nu(\nu(i)+p))} \uparrow i & \text { since } i \leq \nu(i)+p \\
& =a^{(\nu(i))} \uparrow i & \text { since } i \leq \nu(i)+p, \text { hence } \nu(i) \leq \nu(\nu(i)+p) \text { by }(12) \\
& =c^{(i)} . &
\end{array}
$$

So we have $s \simeq \pi(s)$, since for any $k, p$, we have proved $a^{(\nu(i)+k)}\left\lceil i=c^{(\nu(i)+p)}\lceil i\right.$.

Let now $s^{\prime}=\left\langle d^{(i)}\right\rangle_{i \in \omega}$ be another projective sequence such that $s \simeq s^{\prime}$. Then it follows $\pi(s) \simeq s^{\prime}$, being $\simeq$ an equivalence relation. So, for any $i \in \omega$, choose $j(i)$ such that $j(i) \geq i$ and for any $p, k \in \omega$, $c^{(j(i)+k)} \uparrow i=d^{(j(i)+p)} \uparrow i$. By Proposition 4.4(1) we obtain $c^{(i)}=d^{(i)}$, hence we have the thesis.

It is known from the works of Walters $[28,29]$ that, in general, the sheaf condition on $\mathbf{A}$, namely the existence of least upper bounds of compatible subsets (that is chains) of $\mathbf{A}$, amounts to Cauchy completeness (see also [30,31]). We can now prove this in an elementary way, as a consequence of the fact that the three notion of completeness for a tiered object coincide.

Proposition 4.6. For a pointed tiered object $\mathbf{A}$ the following are equivalent:

1. A is projectively complete;

2. A is Cauchy complete; 
3. A is complete.

\section{Proof:}

We will prove $(1) \Rightarrow(2) \Rightarrow(3) \Rightarrow(1)$.

$(1) \Rightarrow(2)$ Let $\mathbf{A}$ be projectively complete, and let $s$ be a Cauchy sequence. Consider the projective sequence $\pi(s)$ defined in Lemma 4.5. Let $a$ be its projective limit. By Proposition 4.4(6) $a$ is also the limit of $\pi(s)$. Since $s \simeq \pi(s)$, we have that $a$ is also the limit of $s$, proving that $\mathbf{A}$ is Cauchy complete.

(2) $\Rightarrow$ (3) Let $\mathbf{A}$ be Cauchy complete. Let $B$ a chain in $\mathbf{A}$. We have to prove that $B$ has a least upper bound. If $B$ is empty or finite the thesis is immediate: $\perp$ or the greatest element of $B$ respectively is the least upper bound. Otherwise, define a sequence $s=\left\langle a^{(n)}\right\rangle_{n \in \omega}$. Let $b_{0} \in B$ arbitrary. Then

$$
\begin{aligned}
a^{(0)} & =b_{0} \\
a^{(n+1)} & =\text { any } b \in B \text { such that } \mathbf{E} b>\mathbf{E} a^{(n)} .
\end{aligned}
$$

Note that $a^{(n+1)}$ is defined, by Proposition 2.7(10), and that for any $b \in B$ there exists $n(b) \in \omega$ such that $b \sqsubseteq a^{(n(b))}$ (take $n(b)$ large enough so that $\mathbf{E} b \leq \mathbf{E} a^{(n(b))}$ and use the fact that $B$ is a chain). Moreover $a^{(n)} \sqsubseteq a^{(n+1)}$, since $B$ is a chain and $\mathbf{E} a^{(n)}<\mathbf{E} a^{(n+1)}$. By induction we have $a^{(n)} \sqsubseteq a^{(n+k)}$ for any $n, k \in \omega$. The sequence $s$ is Cauchy. In fact, by the last statement above and Proposition 2.7(5), $a^{(i)} \uparrow i \sqsubseteq a^{(i+k)} \uparrow i$, for any $i \in \omega$. Now observe that by definition, for any $i \in \omega$, we have $\mathbf{E} a^{(i)} \geq i$. Therefore it follows

$$
\begin{array}{rlr}
\mathbf{E}\left(a^{(i)} \uparrow i\right) & =\mathbf{E}\left(a^{(i)}\right) \wedge i & \\
& =i & \\
& =\mathbf{E}\left(a^{(i+k)}\right) \wedge i & \text { by the remark above } \\
& =\mathbf{E}\left(a^{(i+k)} \uparrow i\right) . & \text { as above }
\end{array}
$$

as above

This implies $s$ is Cauchy (by choosing $j(i)=i$ in equation (7)). Let $a^{(\infty)}$ be the limit of $s$. We prove that $a^{(\infty)}$ is the least upper bound of $B$. Given $b \in B$, consider the element $a^{(n(b))}$ defined above. Let $m=\mathbf{E} a^{(n(b))}$. Then by definition of limit, there exists $j(m) \in \omega$ such that for all $k \in \omega$, $a^{(\infty)}\left\lceil m=a^{(j(m)+k)}\left\lceil m\right.\right.$. On the other hand $a^{(j(m)+k)} \uparrow m=a^{(n(b))}$, since $a^{(n(b))} \sqsubseteq a^{(j(m)+k)}$, hence we have $a^{(\infty)} \uparrow m=a^{(n(b))}$, which proves $a^{(n(b))} \sqsubseteq a^{(\infty)}$. Now we conclude $b \sqsubseteq a^{(\infty)}$ by transitivity. Therefore we have proved that $a^{(\infty)}$ is an upper bound for $B$.

For uniqueness, assume that $u$ is any upper bound of $B$, then $u$ is also an upper bound of $s=$ $\left\langle a^{(n)}\right\rangle_{n \in \omega}$ because any $a^{(i)} \in B$. Then, for any $i \in \omega$ we have:

$$
\begin{aligned}
u \uparrow i & =u \uparrow\left(\mathbf{E} a^{(i)} \wedge i\right) \\
& =\left(u \uparrow \mathbf{E} a^{(i)}\right) \uparrow i \\
& =a^{(i)} \uparrow i \\
& =a^{(i+k)} \uparrow i
\end{aligned}
$$$$
\text { because } \mathbf{E} a^{(i)} \geq i
$$$$
\text { because } u \text { upper bound of } s
$$

for any $k \in \omega$, by the proof that $s$ is Cauchy.

Hence $u$ is a limit for $s$ and then it must coincide with $a^{(\infty)}$.

(3) $\Rightarrow$ (1) Let $\mathbf{A}$ be complete. Let $s=\left\langle a^{(i)}\right\rangle_{i \in \omega}$ a projective sequence. Then $s$ is an ascending chain, 
by Proposition 4.4(2). Let $a=\bigsqcup s$. Then $a$ is also the projective limit of $s$, since for any $n \in \omega$

$$
\begin{aligned}
a\lceil n & =(\bigsqcup s)\lceil n \\
& =\bigsqcup_{i \in \omega}\left(a^{(i)}\lceil n)\right. \\
& =a^{(n)}
\end{aligned}
$$

by Lemma $2.10(3)$

by Proposition 4.4(1).

\section{Projective completion of tiered objects}

Two standard completions are possible for tiered object: ideal completion or metric completion, according to whether we choose the partial order or the metric as the tool for the completion process. For a comparison between the two approaches see [32]. In general, the two completions do not coincide. Anyway tiered objects afford a simpler kind of completion, based on projective sequences, which we call projective completion. The projective completion of a tiered object is a new tiered object isometric to the standard metric completion: we just state this result without proving it, since it will not be used in the remainder of the paper. Instead we will prove that the projective completion and the ideal completion of a finitary tiered object are isomorphic: this result is in order, since it connects tiered objects and their projective completions with the results of Adámek [15]. In Section 7.1 we will see an application of this fact. Projective completion is based on the property of a tiered object shown in Lemma 4.5: for any Cauchy sequence there exists a unique projective sequence $\simeq$-related to it: this allows to choose projective sequences as the canonical representatives for all Cauchy sequences. As a consequence, the completion process is carried out by simply taking the points of finite extent together with the projective sequences, without any need of quotienting the sequences (see [33], Chapter 6). As a further consequence, the embedding of a finitary tiered object in its projective completion is set-theoretic inclusion.

We start with the definition of finitary tiered object.

Definition 5.1. 1. Given a tiered object $\mathbf{A}$, let $A^{*}={ }_{\text {def }}\{a \in A \mid \mathbf{E} a \in \omega\}$. $\mathbf{A}^{*}$ is defined as the tiered object whose underlying set is $A^{*}$, with restriction and extent induced by $\mathbf{A}$.

2. A tiered object $\mathbf{A}$ is finitary if $\mathbf{A}=\mathbf{A}^{*}$.

Remark 5.2. Observe that any projective sequence is formed by elements in $A^{*}$.

We now define the projective completion. The standard Cauchy completion (see for instance [33], Chapter 6), is based on taking $\simeq$-equivalence classes of Cauchy sequences. Tiered objects allow to choose canonical representatives of such classes, namely the projective sequences $\pi(s)$ of Lemma 4.5. This simplifies the construction of the completion: elements of finite extent are put directly into the projective completion; then we add all the projective sequences.

Definition 5.3. Let $\mathbf{A}=\langle A, \uparrow, \mathbf{E}\rangle$ be a tiered object. Let $\mathcal{C}^{\infty}(\mathbf{A})$ be the set of $\mathbf{E}$-unbounded projective sequences. Define the projective completion of $\mathbf{A}, \mathbf{A}^{\infty}=\left\langle A^{\infty}, \uparrow^{\infty}, \mathbf{E}^{\infty}\right\rangle$ by: 


$$
\begin{aligned}
& -A^{\infty}=A^{*} \cup \mathcal{C}^{\infty}(\mathbf{A}) \\
& -x \uparrow^{\infty} p= \begin{cases}x \uparrow p & \text { if } x \in A^{*} \\
a^{(p)} & \text { if } x=\left\langle a^{(n)}\right\rangle_{n \in \omega} \in \mathcal{C}^{\infty}(\mathbf{A}) \text { and } p \in \omega \\
x & \text { if } x \in \mathcal{C}^{\infty}(\mathbf{A}) \text { and } p=\infty\end{cases} \\
& -\mathbf{E}^{\infty} x= \begin{cases}\mathbf{E} x & \text { if } x \in A^{*} \\
\infty & \text { if } x \in \mathcal{C}^{\infty}(\mathbf{A})\end{cases}
\end{aligned}
$$

The following lemma is easily proven.

Lemma 5.4. If $\mathbf{A}$ is pointed, then $\mathbf{A}^{\infty}$ is a complete tiered object.

\section{Proof:}

We just prove that $\mathbf{A}^{\infty}$ is complete. By Proposition 4.6 it is enough to prove that $\mathbf{A}^{\infty}$ is projectively complete. Since projective sequences $\left\langle z^{(i)}\right\rangle_{i \in \omega}$ in $\mathbf{A}$ are elements of $\mathbf{A}^{\infty}$, we avoid confusion of notation by using square brackets for projective sequences in $\mathbf{A}^{\infty}$. So, let $s=\left[x^{(i)}\right]_{i \in \omega} \in A^{\infty}$ be a projective sequence. Then, for any $i \in \omega, \mathbf{E}^{\infty} x^{(i)}$ is finite, hence $x^{(i)} \in A^{*}$. On the other hand, by Definition 5.3 it follows immediately $\mathbf{E}^{\infty} x^{(i)}=\mathbf{E} x^{(i)}$. This implies that $s^{\prime}=\left\langle x^{(i)}\right\rangle_{i \in \omega} \subseteq A$ is projective in A. Finally we have $s^{\prime} \uparrow^{\infty} p=x^{(p)}$, which proves that $s^{\prime}$ is the projective limit for $s$ in $\mathbf{A}^{\infty}$.

The next lemma shows that for finitary tiered objects, projective completions are isomorphic to ideal completions, since they satisfy the universal property of ideal completions. This result is simple but important, since it allows to connect the results of the coming sections to Adámek [15]. Moreover, note that the embedding of a finitary $\mathbf{A}$ in its projective completion is given simply by the set-theoretic inclusion.

Lemma 5.5. Let $\mathbf{A}$ be a finitary pointed tiered object. Let $\theta_{\mathbf{A}}: \mathbf{A} \rightarrow \mathbf{A}^{\infty}$ be the inclusion. Then $\theta_{\mathbf{A}}$ is monotone and for any cpo $X$ and monotone function $f: \mathbf{A} \rightarrow X$, there exists a unique continuous extension $f^{+}: \mathbf{A}^{\infty} \rightarrow X$ such that the following diagram commutes:

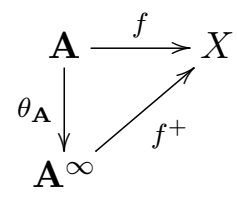

\section{Proof:}

First of all note that it makes sense to speak of continuous functions from $\mathbf{A}^{\infty}$, since by Lemma 5.4 and Corollary $2.11 \mathbf{A}^{\infty}$ is a cpo. The commutativity of the diagram forces the definition of $f^{+}$on $\mathbf{A}$ :

$$
f^{+}\left(\theta_{\mathbf{A}}(a)\right)=f(a)
$$

Now observe that for any sequence $s=\left\langle a^{(n)}\right\rangle_{n \in \omega} \in \mathcal{C}^{\infty}(\mathbf{A}), s=\bigsqcup_{n \in \omega} a^{(n)}$ (the sup is of course taken in $\left.\mathbf{A}^{\infty}\right)$. Then we define $f^{+}$by continuous extension

$$
f^{+}(s)=\bigsqcup_{n \in \omega} f\left(a^{(n)}\right) .
$$




\section{Limits, colimits and tiered objects}

In this section we investigate a particular relation between limits and colimits which arises when we consider sequences of the shape

$$
\begin{aligned}
& \Delta=\left\langle X_{n}, i_{n}: X_{n} \rightarrow X_{n+1}\right\rangle_{n \in \omega}, \\
& \nabla=\left\langle X_{n}, j_{n}: X_{n+1} \rightarrow X_{n}\right\rangle_{n \in \omega} .
\end{aligned}
$$

In general no meaningful relation between $\lim \Delta$ and $\lim \nabla$ holds. But if $\langle\Delta, \nabla\rangle$ is tierable pair (see below Definition 6.1), then $\lim \Delta$ is a (pointed) tiered object whose projective completion is $\lim \nabla$. Conversely, any pointed tiered object $\mathbf{A}$ induces a tierable pair which allows to recover $\mathbf{A}$ (see Theorem 6.13). An interesting application of the results of the present section occurs when considering sequences generated by certain inclusion preserving functors (such as polynomial functors): in such a case final coalgebras arise as projective completions of initial algebras. We will study this case in Section 7.1.

Given $f, g: A \rightarrow B$, let $\mathcal{E}(f, g)=\{a \in A \mid f(a)=g(a)\}$, that is $\mathcal{E}(f, g)$ is the (domain of the) equalizer of $f$ and $g$.

Definition 6.1. Consider two sequences $\Delta=\left\langle X_{n}, i_{n}: X_{n} \rightarrow X_{n+1}\right\rangle$ and $\nabla=\left\langle X_{n}, j_{n}: X_{n+1} \rightarrow\right.$ $\left.X_{n}\right\rangle_{n \in \omega}$. For any $n \in \omega$, let $\mu_{n}: X_{n+1} \rightarrow X_{n+1}$ be defined as $\mu_{n}=i_{n} \circ j_{n}$.

We say that the pair $\langle\Delta, \nabla\rangle$ is tierable if $X_{0}$ is the one-point set and the following properties are satisfied for all $n \in \omega$ :

1. $j_{n} \circ i_{n}=\operatorname{Id}_{X_{n}}$.

2. $\mathcal{E}\left(j_{n+1}, \mu_{n} \circ j_{n+1}\right) \subseteq \mathcal{E}\left(\operatorname{Id}_{X_{n+2}}, \mu_{n+1}\right)$.

In the following, given $p \leq r \in \omega$, define:

$$
\begin{aligned}
i_{p, r} & =i_{r-1} \circ \ldots \circ i_{p}, \\
j_{r, p} & =j_{p} \circ \ldots \circ j_{r-1}, \\
\mu_{r[p]} & =i_{p, r} \circ j_{r, p} .
\end{aligned}
$$

where the empty composition when $p=r$ generates by convention identity functions. Moreover define, for any $n, p$

$$
\vartheta_{n, p}= \begin{cases}i_{n, p} & \text { if } n \leq p \\ j_{n, p} & \text { if } n>p\end{cases}
$$

The proof of the next lemma follows easily from Definition 6.1(1).

Lemma 6.2. Let $\langle\Delta, \nabla\rangle$ be tierable, and let $p \leq r$. Then:

1. $i_{p}$ is mono, $j_{p}$ is epi;

2. $j_{r, p} \circ i_{p, r}=\operatorname{Id}_{X_{p}}$;

3. $\forall n \in \omega \cdot \vartheta_{r, n} \circ i_{p, r}=\vartheta_{p, n}$; 
4. $\forall n \in \omega \cdot j_{r, p} \circ \vartheta_{n, r}=\vartheta_{n, p}$;

5. $\mu_{r[p]} \circ i_{p, r}=i_{p, r}$.

Consider a pair $\langle\Delta, \nabla\rangle$, and suppose that all the conditions of Definition 6.1 hold, except for 6.1(2). Given $x \in X_{r}$, suppose that for some $q<r$ it holds

$$
i_{q} \circ j_{r, q}(x)=j_{r, q+1}(x) .
$$

If we interpret $j_{r, q}(x)$ as the partial information on $x$ available at level $q$, then equality (13) says that level $q$ and level $q+1$ contain the same information on $x$. In general, this stabilization of information does not extend to the following levels $q+2, q+3, \ldots$. But if condition 6.1(2) holds, then the stabilization given by equality (13) is complete: information at level $q$ allows to recover $x: x=$ $i_{q, r} \circ j_{r, q}(x)=\mu_{r[q]}(x)$. What above is the informal meaning of next lemma.

Lemma 6.3. Let $\langle\Delta, \nabla\rangle$ be tierable. Let $h, q, p, r \in \omega$, with $q<p \leq r$. Then

1. $\forall x \in X_{q+2} \cdot j_{q+1}(x) \in i_{q}\left(X_{q}\right) \Rightarrow \mu_{q+1}(x)=x$;

2. $\forall x \in X_{r} . \mu_{p[q]} \circ j_{r, p}(x)=j_{r, p}(x) \Rightarrow \mu_{r[q]}(x)=x$;

3. $\forall x \in X_{r} \cdot \mu_{r[q]}(x)=\mu_{r[p]}(x) \Rightarrow \mu_{r[q]}(x)=x$;

4. Let $\sigma=\left\langle x^{(k)}\right\rangle_{k \in \omega} \in \varliminf_{\lim } \xi . i_{q}\left(x^{(q)}\right)=x^{(q+1)} \Rightarrow \vartheta_{q, h}\left(x^{(q)}\right)=x^{(h)}$.

Moreover, for the points (2) and (3) the reverse implications hold, for any $q \leq p \leq r$.

\section{Proof:}

The reverse implications $(\Leftarrow)$ for points $(2)$ and (3) follow easily from definitions. We prove the $(\Rightarrow)$ implications.

(1) Let $j_{q+1}(x)=i_{q}(y)$, for some $y \in X_{q}$. Then

$$
\begin{aligned}
\mu_{q} \circ j_{q+1}(x) & =\mu_{q} \circ i_{q}(y) \\
& =i_{q}(y) \\
& =j_{q+1}(x) .
\end{aligned}
$$

Therefore $x \in \mathcal{E}\left(j_{q+1}, \mu_{q} \circ j_{q+1}\right)$, and from Definition 6.1(2) it follows $x \in \mathcal{E}\left(\operatorname{Id}_{X_{q+2}}, \mu_{q+1}\right)$, whence the thesis.

(2) We reason by induction on $r-p$. If $r=p$ the thesis is trivial. Otherwise, the hypothesis $\mu_{p[q]} \circ$ $j_{r, p}(x)=j_{r, p}(x)$ can be rephrased as $\mu_{p[q]} \circ j_{r-1, p} \circ j_{r-1}(x)=j_{r-1, p} \circ j_{r-1}(x)$. By induction it follows

$$
\mu_{r-1[q]} \circ j_{r-1}(x)=j_{r-1}(x) .
$$

Equality (14) implies that $j_{r-1} \in i_{r-2}\left(X_{r-2}\right)$. By previous point (1) we have $\mu_{r-1}(x)=x$. Now we have:

$$
\begin{aligned}
\mu_{r[q]}(x) & =i_{r-1} \circ \mu_{r-1[q]} \circ j_{r-1}(x) \\
& =i_{r-1} \circ j_{r-1}(x) \\
& =\mu_{r-1}(x) \\
& =x
\end{aligned}
$$

by above. 
(3)

$$
\begin{aligned}
\mu_{r[q]}(x)=\mu_{r[p]}(x) & \Leftrightarrow i_{q, r} \circ j_{r, q}(x)=i_{p, r} \circ i_{p, r}(x) \\
& \Leftrightarrow i_{p, r} \circ i_{q, p} \circ j_{p, q} \circ j_{r, p}(x)=i_{p, r} \circ i_{p, r}(x) \\
& \Leftrightarrow i_{p, r} \circ \mu_{p[q]} \circ j_{r, p}(x)=i_{p, r} \circ j_{r, p}(x) \\
& \Leftrightarrow \mu_{p[q]} \circ j_{r, p}(x)=j_{r, p}(x) \\
& \Rightarrow \mu_{r[q]}(x)=x
\end{aligned}
$$

(4) Let $h>q$.

$$
\begin{aligned}
i_{q}\left(x^{(q)}\right)=x^{(q+1)} & \Leftrightarrow i_{q+1, h} \circ i_{q}\left(x^{(q)}\right)=i_{q+1, h}\left(x^{(q+1)}\right) \\
& \Leftrightarrow i_{q+1, h} \circ i_{q} \circ j_{h, q}\left(x^{(h)}\right)=i_{q+1, h} \circ j_{h, q+1}\left(x^{(h)}\right) \\
& \Leftrightarrow \mu_{h[q]}\left(\left(x^{(h)}\right)=\mu_{h[q+1]}\left(x^{(h)}\right) .\right.
\end{aligned}
$$

From previous point (3) we have $\mu_{h[q]}\left(x^{(h)}\right)=x^{(h)}$. From definition of $\mu_{h[q]}$ it follows $i_{q, h}\left(x^{(q)}\right)=$ $x^{(h)}$, hence the thesis.

If $h \leq q$ the thesis follows immediately from definition of $\sigma$ and $\vartheta_{q, h}$.

As expected, a pointed tiered object induces a tierable pair.

Definition 6.4. Let $\mathbf{A}=\langle A, \uparrow, \mathbf{E}\rangle$ be a pointed tiered object. We define the sequence $\nabla_{\mathbf{A}}=\left\langle A_{n}, j_{n}\right.$ : $\left.A_{n+1} \rightarrow A_{n}\right\rangle_{n \in \omega}$ as follows:

- $A_{n}=\{a \in A \mid \mathbf{E} a \leq n\}$;

- $\forall a \in A_{n+1} \cdot j_{n}(a)=a\lceil n$.

Finally we define the sequence $\Delta_{\mathbf{A}}=_{\text {def }}\left\langle A_{n}, \subseteq\right\rangle_{n \in \omega}$, and $\alpha_{\mathbf{A}}$ as the pair $\left\langle\nabla_{\mathbf{A}}, \Delta_{\mathbf{A}}\right\rangle$.

Remark 6.5. Let $p \leq r \in \omega$. With reference to Definition 6.4, it holds $j_{r, p}(a)=a\lceil p$. This follows easily from definition of $j_{r, p}$ as composition of $j_{n}$, and from Definition 2.3(2).

Lemma 6.6. $\alpha_{\mathrm{A}}$ is tierable.

\section{Proof:}

We prove that conditions of Definition 6.1 are satisfied.

It is trivial that $A_{0}$ is the one-point set, since $\mathbf{A}$ is pointed. As to Definition 6.1(1), let $a \in A_{n}$. Then

$$
\begin{aligned}
j_{n} \circ i_{n}(a) & =a\lceil n \\
& =a \quad \text { by Proposition 2.7(12), since by definition of } A_{n}, \mathbf{E} a \leq n .
\end{aligned}
$$

As to Definition 6.1(2), let $a \in A_{n+2}$ and $j_{n+1}(a)=\mu_{n} \circ j_{n+1}(a)$. Using Definition 6.4, it follows:

$$
\begin{aligned}
a \uparrow(n+1) & =j_{n+1}(a) \\
& =\mu_{n} \circ j_{n+1}(a) \\
& =i_{n} \circ j_{n} \circ j_{n+1}(a) \\
& =(a\lceil n+1)\lceil n \\
& =a \uparrow(n+1 \wedge n) \\
& =a\lceil n .
\end{aligned}
$$


By Proposition 2.7(7) it follows $\mathbf{E} a \leq n$. Therefore we have

$$
\begin{aligned}
a & =a\lceil n+1 \\
& =j_{n+1}(a) \\
& =\mu_{n+1}(a)
\end{aligned}
$$

by Proposition 2.7(12)

since $i_{n+1}$ is an inclusion.

Now we consider the opposite direction: defining tiered objects out of tierable pairs. We recall the standard definition of $\omega$-colimits in the category of sets, adapted to our framework (see Theorem 1 in Chapter IX, Section 2 of [34]).

Lemma 6.7. Let $\Delta=\left\langle X_{n}, i_{n}: X_{n} \rightarrow X_{n+1}\right\rangle_{n \in \omega}$. Define $D^{+}=\bigcup_{q \in \omega}\left(X_{q} \times\{q\}\right)$, and $D_{\Delta}=$ $D^{+} / \sim$, where $\sim$ is the equivalence relation defined by

$$
\begin{aligned}
\langle x, q\rangle \sim\langle y, p\rangle \Leftrightarrow \quad & q \leq p \& y=i_{q, p}(x) \text { or } \\
& p<q \& x=i_{p, q}(y)
\end{aligned}
$$

1. Let $v_{q}: X_{q} \rightarrow D_{\Delta}$ defined by $v_{q}(x)=[\langle x, q\rangle]_{\sim}$, for any $x \in X_{q}$. Then $\left\langle D_{\Delta},\left\langle v_{q}\right\rangle_{q \in \omega}\right\rangle$, is the colimit of $\Delta$.

2. Let $q \leq r \in \omega, x \in X_{q}$. Then $\langle x, q\rangle \sim\left\langle i_{q, r}(x), r\right\rangle$.

3. Let $w_{q}: D_{\Delta} \rightarrow X_{q}$ be defined by $w_{q}(\bar{u})=\vartheta_{p, q}(x)$, for any $\bar{u}=[\langle x, p\rangle]_{\sim} \in D_{\Delta}$. Then $w_{q}$ is well-defined.

\section{Proof:}

We just prove the last statement. Suppose that both $\bar{u}=[\langle x, p\rangle]_{\sim}$ and $\bar{u}=[\langle y, r\rangle]_{\sim}$ holds, with $p \leq r$. Then $y=i_{p, r}(x)$, hence $\vartheta_{r, q}(y)=\vartheta_{r, q}\left(i_{p, r}(x)\right)=\vartheta_{p, q}(x)$, by Lemma 6.2(3). Therefore $w_{q}(\bar{u})$ is independent from the choice of the element chosen in $\bar{u}$.

In the following we will omit the subscript $\sim$ in the notation for the equivalence classes.

Definition 6.8. Let $\alpha=\langle\Delta, \nabla\rangle$ be tierable. We define a structure $\mathbf{D}_{\alpha}=\left\langle D_{\alpha}, \uparrow_{\alpha}, \mathbf{E}_{\alpha}\right\rangle$ as follows:

- $D_{\alpha}=D_{\Delta}$.

- for any pair $\langle x, q\rangle \in D^{+}$, let $\mathcal{M}_{\langle x, q\rangle}=\left\{k \mid k \leq q \wedge \mu_{q[k]}(x)=x\right\}$. Let $\bar{u}=[\langle x, q\rangle] \in D_{\alpha}$. Define:

$$
\begin{aligned}
& \text { - } \quad * \forall n \in \omega \cdot \bar{u} \uparrow_{\alpha} n=\left[\left\langle\vartheta_{q, n}(x), n\right\rangle\right] ; \\
& \quad * \bar{u} \uparrow_{\alpha} \infty=\bar{u} . \\
& \text { - } \mathbf{E}_{\alpha} \bar{u}=\min \mathcal{M}_{\langle x, q\rangle} .
\end{aligned}
$$

Lemma 6.9. Let $\alpha$ be tierable. Then the restriction $\uparrow_{\alpha}$ and the extent $\mathbf{E}_{\alpha}$ are well-defined. 


\section{Proof:}

Let $\bar{u} \in D_{\alpha}$. We prove that restriction and extent produce the same results, regardless of the choice of $\langle x, q\rangle,\langle y, p\rangle$ such that $[\langle x, q\rangle]=\bar{u}=[\langle y, p\rangle]$. Without loss of generality, let $q \leq p$, so we have $y=i_{q, p}(x)$.

First we consider the restriction. If $n=\infty$, then $\left([\langle x, q\rangle] \uparrow_{\alpha} n\right)=\bar{u}=\left([\langle y, p\rangle] \uparrow_{\alpha} n\right)$ follows immediately from Definition 6.8. Let $n \in \omega$. We have:

$$
\begin{aligned}
{[\langle x, q\rangle] \Gamma_{\alpha} n } & =\left[\left\langle\vartheta_{q, n}(x), n\right\rangle\right] \\
& =\left[\left\langle\vartheta_{p, n} \circ i_{q, p}(x), n\right\rangle\right] \\
& =\left[\left\langle\vartheta_{p, n}(y), n\right\rangle\right] \\
& =[\langle y, p\rangle] \uparrow_{\alpha} n .
\end{aligned}
$$

This completes the proof for $\uparrow_{\alpha}$.

As to $\mathbf{E}_{\alpha}$, let $k \leq q$. We prove that for any $k \leq q, k \in \mathcal{M}_{\langle x, q\rangle}$ if and only if $k \in \mathcal{M}_{\langle y, p\rangle}$. In fact we have

$$
\begin{aligned}
& \mu_{q[k]}(x)=x \Leftrightarrow \mu_{q[k]} \circ j_{p, q}(y)=j_{p, q}(y) \\
& \text { using } i_{q, p}(x)=y \\
& \Leftrightarrow i_{q, p} \circ \mu_{q[k]} \circ j_{p, q}(y)=\mu_{p[q]}(y) \quad \text { composing with and using injectivity of } i_{q, p} \\
& \Leftrightarrow \mu_{p[k]}(y)=\mu_{p[q]}(y) \\
& \Leftrightarrow \mu_{p[k]}(y)=\mu_{p[q]} \circ i_{q, p}(x) \quad \text { using } i_{q, p}(x)=y \\
& \Leftrightarrow \mu_{p[k]}(y)=i_{q, p}(x) \\
& \Leftrightarrow \mu_{p[k]}(y)=y \text {. }
\end{aligned}
$$

As a consequence, $\min \mathcal{M}_{\langle x, q\rangle}=\min \mathcal{M}_{\langle y, p\rangle}$, therefore $\mathbf{E}_{\alpha} \bar{u}$ is well-defined.

Lemma 6.10. Given a tierable $\alpha=\langle\Delta, \nabla\rangle, \mathbf{D}_{\alpha}$ is a finitary pointed tiered object.

\section{Proof:}

The unique point in $X_{0}$ is the bottom element.

We prove that the three conditions of Definition 2.3 hold.

Let $\bar{u}=[\langle x, r\rangle]$, and $\mathbf{E}_{\alpha} \bar{u}=k$. Then, by definition of $\mathbf{E}_{\alpha}, k \leq r$ and $\mu_{r[k]}(x)=x$, which implies

$$
\left\langle j_{r, k}(x), k\right\rangle \sim\langle x, r\rangle \text {. }
$$

Therefore we have:

$$
\begin{aligned}
\bar{u} \uparrow_{\alpha} \mathbf{E}_{\alpha} \bar{u} & =[\langle x, r\rangle] \uparrow_{\alpha} k \\
& =\left[\left\langle j_{k, r}(x), k\right\rangle\right] \\
& =[\langle x, r\rangle] \\
& =\bar{u}
\end{aligned}
$$

Therefore condition (1) of Definition 2.3 holds.

Consider now condition (2) of Definition 2.3. In what follows we will omit the subscript of the 
restriction $\uparrow_{\alpha}$. Let $\bar{u}$ as above, and $q<p \in \omega$. Any case for which $q>r$ or $p>r$ is trivial. So let $q, p \leq r$. We prove that $(\bar{u}\lceil q) \uparrow p=(\bar{u} \uparrow p) \uparrow q=\bar{u} \uparrow q$. In fact

$$
\begin{aligned}
(\bar{u}\lceil q) \uparrow p & =\left[\left\langle j_{r, q}(x), q\right\rangle\right]\lceil p \\
& =\left[\left\langle j_{r, q}(x), q\right\rangle\right] .
\end{aligned}
$$

On the other hand

$$
\begin{aligned}
(\bar{u}\lceil p) \uparrow q & =\left[\left\langle j_{r, p}(x), p\right\rangle\right]\lceil q \\
& =\left[\left\langle j_{p, q} \circ j_{r, p}(x), q\right\rangle\right] \\
& =\left[\left\langle j_{r, q}(x), q\right\rangle\right] .
\end{aligned}
$$

We now consider condition (3) of Definition 2.3. The case $p>r$ is trivial, since it yields to

$$
\begin{aligned}
\mathbf{E}_{\alpha}(\bar{u} \uparrow p) & =\mathbf{E}_{\alpha} \bar{u} & \text { since } \bar{u} \uparrow p=\bar{u} \\
& =\mathbf{E}_{\alpha} \bar{u} \wedge p & \text { since } p>r \geq \mathbf{E}_{\alpha} \bar{u} .
\end{aligned}
$$

Consider the case $p \leq r$. We have

$$
\mathbf{E}_{\alpha}\left(\bar{u}\lceil p)=\min \left\{k \leq p \mid \mu_{p[k]} \circ j_{r, p}(x)=j_{r, p}(x)\right\} .\right.
$$

In the following, we abbreviate $\mathbf{E}_{\alpha}\left(\bar{u}\lceil p)\right.$ with $e$. Observe that for any $k \leq p, \mu_{r[k]}(x)=x$ implies $\mu_{p[k]} \circ j_{r, p}(x)=j_{r, p}(x)$, by the reverse implication of Lemma 6.3(2). This fact, along with $e \leq p$ (trivial by definition of $e$ ), implies $e \leq \mathbf{E}_{\alpha} \bar{u} \wedge p$.

For proving $e \geq \mathbf{E}^{\alpha} \bar{u} \wedge p$, we consider two cases: if $e \geq p$, the thesis is trivial. If $e<p$, we observe that, by definition of $e, \mu_{p[e]} \circ j_{r, p}(x)=j_{r, p}$, hence we can apply Lemma 6.3(2) and derive $\mu_{r[e]}(x)=x$. This last fact implies $\mathbf{E}_{\alpha} \bar{u} \leq e$, and the thesis follows.

As an immediate consequence of Lemma 5.4 we have

Corollary 6.11. Given a tierable pair $\alpha, \mathbf{D}_{\alpha}^{\infty}$ is a complete tiered object.

Remark 6.12. The constructions defined in Lemma 6.7 and Definition 6.8 simplify when, given a pair $\alpha=\langle\nabla, \Delta\rangle$, all the $i_{q}$ 's in the sequence $\Delta=\left\langle X_{q}, i_{q}: X_{q} \rightarrow X_{q+1}\right\rangle_{q \in \omega}$ are set-theoretic inclusions. In such a case we just define:

$$
\begin{aligned}
D_{\Delta} & =\bigcup_{q \in \omega} X_{q} . \\
\forall x \in D_{\Delta} \cdot x \uparrow_{\alpha} q & = \begin{cases}j_{r, q}(x) & \text { if } x \in X_{r}, q \leq r ; \\
x & \text { if } x \in X_{r}, q>r .\end{cases} \\
\forall x \in D_{\Delta} . \mathbf{E}_{\alpha} x & =\min \left\{k \mid x \in X_{k}\right\} . \\
\forall x \in X_{q} \cdot v_{q}(x) & =x . \\
\forall x \in D_{\Delta} \cdot w_{q}(x) & =x \uparrow_{\alpha} q .
\end{aligned}
$$

Before exploring the inverse limit behaviour of $D_{\alpha}^{\infty}$, we first show that an expected result holds: the tour

$$
\mathbf{A} \rightarrow \alpha_{\mathbf{A}} \rightarrow \mathbf{D}_{\alpha_{\mathbf{A}}} \rightarrow \mathbf{D}_{\alpha_{\mathbf{A}}}^{\infty}
$$

is circular when $\mathbf{A}$ is a complete tiered object. 
Theorem 6.13. Let $\mathbf{A}$ be a complete tiered object. Then $\mathbf{D}_{\alpha_{\mathbf{A}}}^{\infty} \cong \mathbf{A}$.

\section{Proof:}

We consider the map $\phi: A \rightarrow D_{\alpha_{\mathbf{A}}}^{\infty}$ defined by:

$$
\phi(a)= \begin{cases}a & \text { if } \mathbf{E} a \in \omega \\ \left\langle a\lceil n\rangle_{n \in \omega}\right. & \text { if } \mathbf{E} a=\infty\end{cases}
$$

By completeness of $\mathbf{A}, \phi$ is a bijection. We show that $\phi$ respects restriction and extent. As to restriction, the proof that

$$
\phi(a \uparrow n)=\phi(a) \uparrow_{\alpha}^{\infty} n
$$

is immediate if $n=\infty$. Otherwise let $n \in \omega$. If $\mathbf{E} a=k<n$, then $a\lceil n=a$, by Proposition 2.7(12). On the other hand

$$
\begin{array}{rlr}
\phi(a) \uparrow_{\alpha}^{\infty} n & =a \uparrow_{\alpha}^{\infty} n & \\
& =a \uparrow_{\alpha} n \\
& =a \quad \text { by Remark } 6.12
\end{array}
$$

If $k \geq n$, both the cases $k \in \omega$ and $k=\infty$ yield $\phi(a) \uparrow_{\alpha}^{\infty} n=a \uparrow_{\alpha} n$. Therefore

$$
\begin{aligned}
\phi(a) \uparrow_{\alpha}^{\infty} n & =a \Gamma_{\alpha} n \\
& =j_{k, n}(a) \\
& =a\lceil n
\end{aligned}
$$

by Remark 6.5 .

As to extent, we have to prove $\mathbf{E} a=\mathbf{E}_{\alpha}^{\infty} \phi(a)$. The thesis is trivial when $\mathbf{E} a=\infty$. Otherwise

$$
\begin{aligned}
\mathbf{E}_{\alpha}^{\infty} \phi(a) & =\mathbf{E}_{\alpha}^{\infty} a \\
& =\mathbf{E}_{\alpha} a \\
& =\min \left\{q \mid a \in A_{q}\right\} \\
& =\min \{q \mid \mathbf{E} a \leq q\} \\
& =\mathbf{E} a .
\end{aligned}
$$

Given a tierable pair $\alpha=\langle\nabla, \Delta\rangle, \mathbf{D}_{\alpha}^{\infty}$ is defined after Definition 5.3 and Lemma 6.7 as the completion of the colimit of $\Delta$. On the other hand, it can be viewed as a cone over $\nabla$ : consider the projections $\pi_{n}: D_{\alpha}^{\infty} \rightarrow X_{n}$ defined as:

$$
\forall s \in D_{\alpha}^{\infty} \cdot \pi_{n}(s)=w_{n}\left(s \uparrow_{\alpha}^{\infty} n\right)
$$

More explicitely:

$$
\pi_{n}(s)= \begin{cases}\vartheta_{q, n}(x) & \text { if } s=[\langle x, q\rangle] \\ a^{(n)} & \text { if } s=\left\langle\left[\left\langle a^{(q)}, q\right\rangle\right]\right\rangle_{q \in \omega}\end{cases}
$$

From Lemma 6.7(3) it follows that $\pi_{n}$ are well-defined, and from Lemma 6.2, points (3) and (4), that $\left\langle\mathbf{D}_{\alpha}^{\infty}, \pi_{n}\right\rangle_{n \in \omega}$ is a cone for $\alpha$. Anyway a stronger result holds. 
Theorem 6.14. Let $\alpha=\langle\Delta, \nabla\rangle$ be tierable. Then

$$
\left\langle\mathbf{D}_{\alpha}^{\infty},\left\langle\pi_{n}\right\rangle_{n \in \omega}\right\rangle \cong \lim \nabla .
$$

Proof:

Let $\left\langle C,\left\langle\beta_{n}: C \rightarrow X_{n}\right\rangle_{n \in \omega}\right\rangle$ be another cone for $\nabla$. Then for any $n \in \omega$,

$$
\beta_{n}=j_{n} \circ \beta_{n+1} .
$$

We will prove that for any $c \in C$ there exists a unique $\varphi(c) \in D_{\alpha}^{\infty}$ such that for any $n \in \omega$,

$$
\beta_{n}(c)=\pi_{n}(\varphi(c)) .
$$

For any $c \in C$, let $s_{c}=\left\langle\bar{u}_{n}\right\rangle_{n \in \omega}$, where for any $n \in \omega, \bar{u}_{n}=v_{n}\left(\beta_{n}(c)\right)$. By definition of $v_{n}$, we have $\bar{u}_{n}=\left[\left\langle\beta_{n}(c), n\right\rangle\right] . s_{c}$ is projective in $\mathbf{D}_{\alpha}$. In fact

$$
\begin{aligned}
\bar{u}_{n+1} \Gamma_{\alpha} n & =\left[\left\langle\beta_{n+1}(c), n+1\right\rangle\right] \uparrow_{\alpha} n \\
& =\left[\left\langle j_{n}\left(\beta_{n+1}(c), n\right\rangle\right]\right. \\
& =\left[\left\langle\beta_{n}(c), n\right\rangle\right] \\
& =\bar{u}_{n} .
\end{aligned}
$$

If $s_{c}$ is E-unbounded, then we choose $\varphi(c)=s_{c}$. In fact, in this case $s_{c} \in D_{\alpha}^{\infty}$, and it is the unique element such that for any $n \in \omega$, it satisfies equality (17) by construction. In fact:

$$
\begin{aligned}
\pi_{n}(\varphi(c)) & =\pi_{n}\left(\left\langle\left[\left\langle\beta_{q}(c), q\right\rangle\right]\right\rangle_{q \in \omega}\right) \\
& =\beta_{n}(c) .
\end{aligned}
$$

If $s_{c}$ is E-bounded, let $k$ be the least $n \in \omega$ such that $\bar{u}_{n}=\bar{u}_{n+1}$, or equivalently $i_{n}\left(\beta_{n}(c)\right)=\beta_{n+1}(c)$. In this case we define $\varphi(c)=\bar{u}_{k}$. We have, for any $n \in \omega$,

$$
\begin{aligned}
\pi_{n}(\varphi(c)) & =\vartheta_{k, n}\left(\beta_{k}(c)\right) & \text { by definition of } \pi_{n} \\
& =\beta_{n}(c) & \text { by Lemma 6.3(4), since } i_{k}\left(\beta_{k}(c)=\beta_{k+1}(c) .\right.
\end{aligned}
$$

Therefore $\varphi(c)$ satisfies equality (17). Now we prove that $\varphi(c)$ is the unique element in $D_{\alpha}^{\infty}$ for which equality (17) holds. Let $\bar{u}^{\prime}=[\langle z, h\rangle] \in D_{\alpha}^{\infty}$ such that for any $n \in \omega, \pi_{n}\left(\bar{u}^{\prime}\right)=\beta_{n}(c)$. In particular we have

$$
\vartheta_{h, k}(z)=\pi_{k}\left(\bar{u}^{\prime}\right)=\beta_{k}(c) .
$$

It is not possible to have $h<k$, since from equation (18) it would follow $z=j_{h, k}\left(\beta_{k}(c)\right)=\beta_{h}(c)$. This would imply $i_{h, k}\left(\beta_{h}(c)\right)=i_{h, k}(z)=\beta_{k}(c)$, against the minimality of $k$. So we have $h \geq k$. On the other hand we have proven above $\vartheta_{k, n}\left(\beta_{k}(c)\right)=\beta_{n}(c)$, for any $n \in \omega$. Choosing $n=h$, we get

$$
\begin{aligned}
i_{k, h}\left(\beta_{k}(c)\right) & =\beta_{h}(c) & \\
& =\pi_{h}\left(\bar{u}^{\prime}\right) & \\
& =z & \text { by definition of } \pi_{h}
\end{aligned}
$$

Therefore we have proven $\left\langle\beta_{k}(c), k\right\rangle \sim\langle z, h\rangle$, hence $\bar{u}^{\prime}=\bar{u}$. 


\section{Applications}

\subsection{Tiered (co)algebras}

Consider the functor $F(X)=\{\epsilon\}+\{0,1\} \times X$. Its final coalgebra is the set of finite and infinite binary sequences, which is isomorphic to the ideal completion (under prefix ordering) of its initial algebra, consisting of the the set of finite binary sequences. A general account of this phenomenon is given by Adámek [15]: under certain hypotheses on functors $F$ : Set $\rightarrow$ Set, final $F$-coalgebras are isomorphic to ideal completions of initial $F$-algebras. In this section we apply the results of Section 6 , and construe final coalgebras $\mathcal{Y}$ as projective completions of initial algebras $\mathcal{X}$, looking at the latter as finitary tiered objects,

$$
\mathcal{Y} \cong \mathcal{X}^{\infty} .
$$

This result is a specialization of the theory of Adámek because, despite our use of projective instead of ideal completion, Lemma 5.5 shows that the two kinds of completion yield isomorphic cpos in the case of finitary tiered objects. Therefore, equation (19) (which summarizes Theorem 7.4 and Corollary 7.5) may not be considered a theoretic improvement of [15]. Nevertheless we believe to be of some interest to expose the details, as the use of projective completion substantiates in a direct way the naive intuition that in many cases final coalgebras can be defined in a set-theoretic way out of initial algebras, by taking all the points in the initial algebras (the "finite points") and adding the projective sequences (the "infinite points").

Definition 7.1. Let $F:$ Set $\rightarrow$ Set a functor. We say that $F$ is tiering if the following conditions hold:

1. $F$ preserves inclusions;

2. $F(\emptyset) \neq \emptyset$;

3. $F$ respects equalizers in the following sense: for every $f, g: Y \rightarrow Y^{\prime}, \mathcal{E}(F(f), F(g)) \subseteq$ $F(\mathcal{E}(f, g)){ }^{1}$

4. for any $f: Y \rightarrow Y^{\prime}, F(f)^{-1}(F(\emptyset)) \subseteq F(\emptyset)$.

As examples of tiering functors, we mention those polynomial functors $F$ which have the shape $F(X)=A+\ldots$, where $A$ is a fixed non-empty set. Consider for instance the polynomial functor $F(X)=A+(B \times X)$, where $A$ and $B$ are fixed sets. It is easy to prove that (1), (2) and (3) are satisfied. Moreover, (4) holds, since given a morphism $f: X \rightarrow Y, F(\emptyset)=A$ and we have that $(F(f))^{-1}(A)=A$. These arguments apply to any polynomial functor.

As an example of non-tiering functor, we mention the finite powerset functor: for instance, condition 7.1(3) does not hold.

Let $F$ be a $\omega$-continuous and $\omega$-cocontinuous tiering functor on Set. Let $d_{0}$ any element of $F(\emptyset)$ : we define $X_{0}=\left\{d_{0}\right\} . X_{0}$ is a terminal object in Set and enjoys the property $X_{0} \subseteq F\left(X_{0}\right)$, since $\emptyset \subseteq X_{0}$ implies $F(\emptyset) \subseteq F\left(X_{0}\right)$ and moreover $X_{0} \subseteq F(\emptyset)$ by definition. Therefore we can build the sequence $\Delta_{F}=\left\langle F^{n}\left(X_{0}\right), \subseteq\right\rangle_{n \in \omega}$.

\footnotetext{
${ }^{1}$ The inclusion makes sense by previous point (1), since both $\mathcal{E}(F(f), F(g))$ and $F(\mathcal{E}(f, g))$ are subset of $F(Y)$.
} 
Remark 7.2. The chain of inclusions

$$
\emptyset \subseteq X_{0} \subseteq F(\emptyset) \subseteq F\left(X_{0}\right) \subseteq F^{2}(\emptyset) \subseteq F^{2}\left(X_{0}\right) \ldots
$$

shows that the colimit of $\Delta_{F}$, namely $\mathbf{D}_{\Delta_{F}}=\bigcup_{n \in \omega} F^{n}\left(X_{0}\right)$, is the initial algebra of $F$.

On the other hand we define the sequence $\nabla_{F}=\left\langle F^{n}\left(X_{0}\right), F^{n}\left(j_{0}\right): F^{n+1}\left(X_{0}\right) \rightarrow F^{n}\left(X_{0}\right)\right\rangle_{n \in \omega}$, where $j_{0}$ is unique to the terminal object. We will prove that the pair $\alpha_{F}=\left\langle\Delta_{F}, \nabla_{F}\right\rangle$ is tierable, and we will apply the results of the previous section. In particular, it will follow that $\mathbf{D}_{\alpha_{F}}$ is the initial algebra by Lemma 6.7(1) and the remark above. On the other hand, its projective completion, namely $\mathbf{D}_{\alpha_{F}}^{\infty}$, is $\lim _{\longleftarrow} \nabla_{F}$ by Theorem 6.14 , hence it is the final coalgebra for $F$, since $X_{0}$ is a terminal object.

Lemma 7.3. Let $F$ be a tiering functor over Set. Let $X_{0} \subseteq F^{n}(\emptyset)$ be a one-point set. With the notation above, $\alpha_{F}$ is tierable.

\section{Proof:}

We prove that condition (1) of Definition 6.1 holds. Exploiting the fact that $i_{n}$ are inclusions, this amounts to show that $\forall n \in \omega \cdot x \in X_{n} \Longrightarrow j_{n}(x)=x$. We reason by induction on $n$. We have $j_{0}\left(d_{0}\right)=d_{0}$, hence the thesis holds for $n=0$. Observe that the following diagram is commutative:

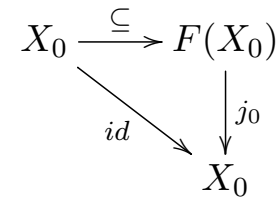

As a consequence, for any $n \in \omega$, the following diagram is commutative too:

$$
\begin{aligned}
& X_{n}=F^{n}\left(X_{0}\right) \stackrel{\subseteq}{\longrightarrow} F^{n+1}\left(X_{0}\right)=X_{n+1}
\end{aligned}
$$

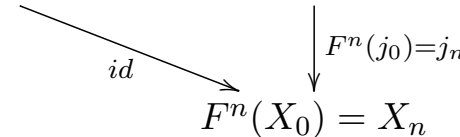

Now we prove that condition (2) of Definition 6.1 holds, that is for any $n \in \omega, \mathcal{E}\left(j_{n+1}, \mu_{n} \circ j_{n+1}\right) \subseteq$ $\mathcal{E}\left(\operatorname{Id}_{X_{n+2}}, \mu_{n+1}\right)$. Taking into account that $i_{n}$ are inclusions, the condition above can be rephrased as:

$$
\mathcal{E}\left(j_{n+1}, j_{n} \circ j_{n+1}\right) \subseteq \mathcal{E}\left(\operatorname{Id}_{X_{n+2}}, j_{n+1}\right) .
$$

We reason by induction. Let $n=0$. Observe that the commutativity of any diagram

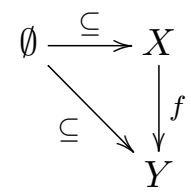

implies the commutativity of

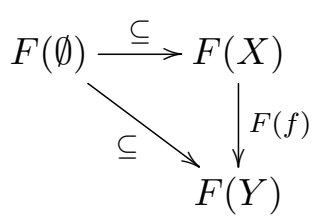


This last diagram implies that $F(f)_{\mid F(\emptyset)}$ behaves as the identity.

Suppose that for some $x, j_{1}(x)=j_{0}\left(j_{1}(x)\right)$. Then $j_{1}(x)=d_{0}$, hence $x \in F\left(j_{0}\right)^{-1}(F(\emptyset))$. Вy Definition 7.1(4), it follows $x \in F(\emptyset)$. Therefore, applying the commutativity of diagram (21) we have $F\left(j_{0}\right)(x)=x$, that is $j_{1}(x)=x$. This proves inclusion (20) for $n=0$.

Now suppose the thesis holds for $n$. We have

$$
\begin{aligned}
\mathcal{E}\left(j_{n+1}, j_{n} \circ j_{n+1}\right) & =\mathcal{E}\left(F\left(j_{n}\right), F\left(j_{n-1} \circ j_{n}\right)\right) \\
& \subseteq F\left(\mathcal{E}\left(j_{n}, j_{n-1} \circ j_{n}\right)\right) \\
& \subseteq F\left(\mathcal{E}\left(\operatorname{Id}_{X_{n+1}}, j_{n}\right)\right) \\
& \subseteq \mathcal{E}\left(F\left(\operatorname{Id}_{X_{n+1}}\right), F\left(j_{n}\right)\right) \\
& =\mathcal{E}\left(\operatorname{Id}_{X_{n+2}}, j_{n+1}\right) .
\end{aligned}
$$

by induction and Definition 7.1(1)

since $F$ is a functor

Theorem 7.4. Let $F:$ Set $\rightarrow$ Set be a $\omega$-continuous tiering functor. Then the final coalgebra is the complete tiered object $\mathbf{D}_{\alpha_{F}}^{\infty}$.

\section{Proof:}

Since $X_{0}$ is chosen to be a terminal object, the final coalgebra is $\lim \nabla_{F}$. By Lemma $7.3 \alpha_{F}$ is tierable, hence $\mathbf{D}_{\alpha_{F}}^{\infty}$ is a complete tiered object, and by Theorem 6.14 it is the limit of $\nabla_{F}$.

Corollary 7.5. Let $F$ : Set $\rightarrow$ Set be a $\omega$-continuous and $\omega$-cocontinuous tiering functor. Then it is possible to define the final coalgebra $\mathcal{Y}$ as the projective completion of the initial algebra $\mathcal{X}$, so that $\mathcal{X} \subseteq \mathcal{Y}$.

\section{Proof:}

By Lemma 6.7(1) $\mathbf{D}_{\alpha_{F}}$ is a colimit for $\left\langle F^{n}\left(X_{0}\right), \subseteq\right\rangle_{n \in \omega}$, which coincides with the initial algebra by Remark 7.2, and by the previous theorem $\mathbf{D}_{\alpha_{F}}^{\infty}$ is the final coalgebra. So the thesis follows from the very definition of $\mathbf{D}_{\alpha_{F}}^{\infty}$ as projective completion of $\mathbf{D}_{\alpha_{F}}$.

\subsection{A proof principle for tiered objects}

We show now how the properties of (complete) tiered objects can be exploited in justifying a general proof principle for their elements. We shall see that the approximation structure given by tiering allows to resolve the apparent circularity implicit in some natural approaches to proving properties of infinite objects.

Properties of elements of a tiered object $\mathbf{A}$ shall be identified with subsets of $\mathbf{A}$ enjoying some simple closure properties that have been suggested by the treatment of the interpretation of recursive types (which has been the initial motivation for the present work) [35, Chapter 10].

Definition 7.6. A subset $P \neq \emptyset$ of a complete tiered objects $\mathbf{A}$ is complete and uniform if it satisfies the following two properties:

Uniformity if $a \in P$ then $a\lceil p \in P$ for all $p \in \Omega$,

Completeness if $\left\{a^{(i)}\right\}_{i \in \omega} \subseteq P$ is an $\sqsubseteq$-increasing chain, then $\bigsqcup_{i \in \omega} a^{(i)} \in P$. 
If $P \subseteq \mathbf{A}$ is complete and uniform, define

$$
\llbracket a \in P \rrbracket=_{\operatorname{def}} \bigvee\{p \in \Omega \mid a\lceil p \in P\} .
$$

By Proposition 2.9(3), we have

$$
p \leq \llbracket a \in P \rrbracket \Leftrightarrow a \uparrow p \in P .
$$

Complete and uniform relations over complete tiered objects can be defined as complete and uniform sets of tuples (that however do not belong to products as characterized, e.g., in [12, §4.8(iv)]):

Definition 7.7. Given complete tiered objects $\mathbf{A}, \mathbf{B}, \mathbf{A} \cdot \mathbf{B}$ is defined as the product $A \times B$ with $\mathbf{E}\langle a, b\rangle=\mathbf{E} a \vee \mathbf{E} b$ and $\langle a, b\rangle \uparrow p=\langle a \uparrow p, b \uparrow p\rangle$.

It is straightforward to show that $\mathbf{A} \cdot \mathbf{B}$ is a complete tiered object. In particular, the diagonal $\Delta_{\mathbf{A}}={ }_{\text {def }}$ $\{\langle a, a\rangle \mid a \in A\}$ is a complete and uniform subset of $\mathbf{A} \cdot \mathbf{A}$, so we can define an $\Omega$-valued equivalence

$$
\begin{aligned}
\llbracket a \equiv b \rrbracket & ={ }_{\operatorname{def}} \llbracket\langle a, b\rangle \in \Delta_{\mathbf{A}} \rrbracket \\
& =\bigvee\{p \in \Omega \mid a\lceil p=b\lceil p\} .
\end{aligned}
$$

Then we have the following consequence of (22):

$$
p \leq \llbracket a \equiv b \rrbracket \Leftrightarrow a\lceil p=b\lceil p .
$$

The following notion of operation and its characterization in terms of restriction are borrowed from $[12,5.1,5.4]$ :

Definition 7.8. Given tiered objects $\mathbf{A}, \mathbf{B}$, an operation is any mapping $f: A \rightarrow B$ such that

$$
\llbracket a \equiv b \rrbracket \leq \llbracket f(a) \equiv f(b) \rrbracket .
$$

Proposition 7.9. For complete tiered objects $\mathbf{A}, \mathbf{B}, f: A \rightarrow B$ is an operation if and only if

$$
f(a) \uparrow p=f(a \uparrow p) \uparrow p
$$

for all $p \in \Omega$ and $a \in A$.

An operation $f: A \rightarrow B$ is guarded if

$$
f(a) \uparrow(p+1)=f(a\lceil p) \uparrow(p+1)
$$

for all $p \in \Omega$ and $a \in A$. Assume that $f: A \rightarrow A$ is guarded and $\mathbf{A}$ is complete. Then build inductively the sequence:

$$
\begin{cases}x^{(0)} & =\perp \\ x^{(n+1)} & =f\left(x^{(n)}\right)\lceil(n+1)\end{cases}
$$

For all $i \in \omega, x^{(i)}=x^{(i+1)} \uparrow i$, so $x^{(\infty)}$ exists and satisfies

$$
x^{(\infty)} \uparrow n=x^{(n)} .
$$


For all $n \in \omega$

$$
f\left(x^{(\infty)}\right)\left\lceil n=x^{(\infty)}\left\lceil n=x^{(n)}\right.\right.
$$

therefore $\lim _{i \rightarrow \infty} x_{(i)}$ is the (unique) fixed point of $f$, by the Approximation Lemma 2.12. Summarizing, we have the following version of the Banach fixed point theorem for complete tiered objects, which has been proved several times in the literature in slightly different forms (see for example $[27,7])$ :

Proposition 7.10. If $\mathbf{A}$ is a complete tiered object, every guarded operation $f: A \rightarrow A$ has a unique fixed point.

For guarded operations we also have the following proof principle, that applies to complete and uniform properties:

Proposition 7.11. Assume that $\mathbf{A}$ is a complete tiered object and $f: \mathbf{A} \rightarrow \mathbf{A}$ is a guarded operation. Then, for any complete and uniform $P \subseteq A$ such that $f(P) \subseteq P$ and any $a \in A$ :

$$
(\llbracket f(a) \in P \rrbracket \leq \llbracket a \in P \rrbracket) \Rightarrow a \in P .
$$

\section{Proof:}

Observe that the premise is equivalent to

$$
\forall n \in \omega(n \leq \llbracket f(a) \in P \rrbracket \Rightarrow n \leq \llbracket a \in P \rrbracket) .
$$

which in turn is equivalent, by (22), to

$$
\forall n \in \omega(f(a)\lceil n \in P \Rightarrow a\lceil n \in P),
$$

then use induction on $n \in \omega: a\lceil 0=\perp \in P$, for every $a \in A$. Assume now that $a\lceil n \in P$ : then $f(a\lceil n) \in P$ because $f(P) \subseteq P$, so also $f(a)\lceil(n+1)=f(a\lceil n) \uparrow(n+1) \in P$ by uniformity of $P$ and because $f$ is guarded. Finally, by the premise, $a\left\lceil(n+1) \in P\right.$, therefore $a=\bigsqcup_{n \in \omega}(a\lceil n) \in P$ by completeness of $P$.

The interesting aspect of this result is that the stratification implicit in the premise of (25) is essential to prove that $a \in P$ (and therefore also that $f(a) \in P$, as $f(P) \subseteq P$ ). A related use of stratification is exploited in the coinductive proof system for subtyping recursive types described in [17].

Remark 7.12. The proof principle of Proposition 7.11 can also be read as a special form of fixpoint induction [36]: in fact, under the assumptions of (25), the unique fixed point of $f$, which exists by Proposition 7.10, is in $P$. Note, in passing, that in this form the principle and its justification look very similar to the metric coinduction principle of $[37,38]$.

We show now an application of Proposition 7.11 to the proof of the following well-known equality in the theory of lazy lists [39]:

$$
\text { nat }=\operatorname{from}(0) \text {, }
$$


where

$$
\begin{aligned}
\text { nat } & =0: \text { inc }(\text { nat }) \\
\operatorname{inc}(\operatorname{nil}) & =\text { nil } \\
\operatorname{inc}(x: x s) & =x+1: \operatorname{inc}(x s) \\
\operatorname{from}(n) & =n: \operatorname{from}(n+1) .
\end{aligned}
$$

Lazy lists are the completion (defined as in Section 5) of the (finitary) tiered object of finite lists, where restriction and extent are given by the usual take and length functions [16]:

$$
\begin{aligned}
\ell \uparrow 0 & =\mathrm{nil} \\
\mathrm{nil} \uparrow n+1 & =\mathrm{nil} \\
(x: \ell) \uparrow n+1 & =x:(\ell \uparrow n) \\
\mathbf{E}(\mathrm{nil}) & =0 \\
\mathbf{E}(x: \ell) & =1+\mathbf{E}(\ell)
\end{aligned}
$$

It is easy to check that these clauses define a tiering on finite lists, that can easily be lifted to a tiering on lazy lists. Now, for any lazy list $x s$, take $f(x s)=0$ : inc $(x s)$, and observe that this is a guarded operation. In order to apply Proposition 7.11, we have to show that, for all $n \in \omega$ :

$$
[0: \operatorname{inc}(\text { nat })\lceil n+1=0: \operatorname{inc}(\operatorname{from}(0))\lceil n+1] \Longrightarrow[\text { nat }\lceil n+1=\operatorname{from}(0)\lceil n+1]
$$

and the premise is equivalent to

$$
\text { nat } \uparrow n+1=0: \operatorname{inc}(\operatorname{from}(0)) \uparrow n+1
$$

We first prove:

Lemma 7.13. For all $n, p \in \omega$,

$$
\operatorname{inc}(\operatorname{from}(n)) \uparrow p=\operatorname{from}(n+1) \uparrow p .
$$

\section{Proof:}

By induction on $p$. The Lemma is obviously true when $p=0$; assume now that $p>0$ :

$$
\begin{aligned}
\operatorname{inc}(n: \operatorname{from}(n+1)) \uparrow p & \\
& =(n+1: \operatorname{inc}(\operatorname{from}(n+1))) \uparrow p \\
& =n+1:(\operatorname{inc}(\operatorname{from}(n+1))\lceil p-1) \\
& =n+1:(\operatorname{from}(n+2) \uparrow p-1) \\
& =(n+1: \operatorname{from}(n+2)) \uparrow p \\
& =\operatorname{from}(n+1) \uparrow p .
\end{aligned}
$$


Then we calculate:

$$
\begin{aligned}
\text { nat } \uparrow n+1 & = \\
& =0: \operatorname{inc}(\operatorname{from}(0)) \uparrow n+1 \\
& =0:(\operatorname{inc}(\operatorname{from}(0))\lceil n) \\
& =0:(\operatorname{from}(1))\lceil n) \\
& =(0: \operatorname{from}(1))\lceil n+1 \\
& =\operatorname{from}(0) \uparrow n+1 .
\end{aligned}
$$

By Proposition 7.11 we conclude that nat $=$ from $(0)$.

\section{Acknowledgments}

We are grateful to an anonymous referee for a careful reading of a draft of this paper, and for comments leading to several improvements.

\section{References}

[1] Bruce K, Mitchell JC. PER models of subtyping, recursive types and higher-order polymorphism. In: Proceedings of the ACM Fourth Symposium on Principles of Programming Languages. ACM; 1992. p. $316-327$.

[2] Baier C, Majster-Cederbaum ME. Metric Semantics from Partial Order Semantics. Acta Informatica. 1997;34(9):701-735

[3] Kummetz R. From Partial Orders with Projections to Domains. Electronic Notes in Theoretical Computer Science. 1999;20.

[4] Herrlich H, Ehrig H. The construct PRO of projection spaces: Its internal structure. In: Ehrig H, Herrlich H, Kreowski HJ, Preuß G, editors. Categorical Methods in Computer Science - with Aspects from Topology. vol. 393 of Lecture Notes in Computer Science. Berlin: Springer-Verlag; 1989. p. 286-293.

[5] Bergstra JA, Klop JW. An introduction to process algebra. In: Baeten JCM, editor. Applications of Process Algebra. No. 17 in Cambridge Tracts in Theoretical Computer Science. Cambridge University Press; 1990. p. $1-21$.

[6] Kranakis E. Fixed point equations with parameters in the projective model. Information and Computation. 1987;75(3):264 - 288 .

[7] Monteiro L. Semantic domains based on sets with families of equivalences. Electronic Notes in Theoretical Computer Science. 1998;11.

[8] Stoltenberg-Hansen V, Lindström I, Griffor ER. Mathematical Theory of Domains. No. 22 in Cambridge Tracts in Theoretical Computer Science. Cambridge University Press; 1994.

[9] Buchholz W. A term calculus for (co-)recursive definitions on streamlike data structures. Annals of Pure and Applied Logic. 2005;136(1-2):75-90.

[10] Matthews J. Recursive functions defined over coinductive types. In: Bertot Y, Dowek G, Hirschowitz A, Paulin C, Théry L, editors. Proceedings of TPHOL '99. vol. 1690 of Lecture Notes in Computer Science. Berlin: Springer-Verlag; 1999. p. 73-90. 
[11] Bukatin MA, Kopperman R, Matthews SG, Pajoohesh H. Partial Metric Spaces. The American Mathematical Monthly. 2009;116(8):708-718.

[12] Fourman MP, Scott DS. Sheaves and Logic. In: Fourman MP, Mulvey C, Scott DS, editors. Applications of Sheaves. vol. 753 of Lecture Notes in Mathematics. Berlin: Springer-Verlag; 1979. p. 302-401.

[13] Gianantonio PD, Miculan M. Unifying Recursive and Co-recursive Definitions in Sheaf Categories. In: Foundations of Software Science and Computation Structures, 7th International Conference, FOSSACS 2004. vol. 2987 of Lecture Notes in Computer Science. Springer; 2004. .

[14] Barr M. Terminal coalgebras in well-founded set theory. Theoretical Computer Science. 1993;114:299_ 315.

[15] Adámek J. Final coalgebras are ideal completions of initial algebras. Journal of Logic and Computation. $2002 ; 12: 217-242$

[16] Gibbons J, Hutton G. Proof Methods for Corecursive Programs. Fundamenta Informaticae. 2005 April/May;66(4):353-366.

[17] Brandt M, Henglein F. Coinductive axiomatization of recursive type equality and subtyping. Fundamenta Informaticæ. 1998;33:309-338.

[18] Coquand T. Infinite objects in type theory. In: Barendregt H, Nipkow T, editors. Types for Proofs and Programs. vol. 806 of Lecture Notes in Computer Science. Berlin: Springer-Verlag; 1993. p. 62-78.

[19] Horn A. Logic with truth values in a linearly ordered Heyting algebra. Journal of Symbolic Logic. 1969;34(3):395-408.

[20] Goldblatt R. Topoi. The Categorial Analysis of Logic. Amsterdam: North-Holland Co.; 1979.

[21] Simmons H. The Point-Free Approach to Sheafification; 2001. Available at http://www.cs.man.ac.uk/ hsimmons/DOCUMENTS/PAPERSandNOTES/Omegasets.pdf.

[22] Bird RS. Introduction to Functional Programming Using Haskell. Prentice-Hall; 1998.

[23] Hutton G, Gibbons J. The Generic Approximation Lemma. Information Processing Letters. 2001;79(4):197-201.

[24] Dedekind R. Essays on the Theory of Numbers. Open Court Publishing Company; 1901. Translation by W.W. Beman of Stetigkeit und irrationale Zahlen (1872) and Was sind und was sollen die Zahlen? (1888), reprinted 1963 by Dover Press.

[25] Henkin L. On mathematical induction. American Mathematical Monthly. 1960;67:323-338.

[26] Flagg B, Kopperman R. Computational models for ultrametric spaces. Electronic Notes in Theoretical Compututer Science. 1997;6.

[27] Ehrig H, Parisi-Presicce F, Boehm P, Rieckhoff C, Dimitrovici C, Grosse-Rhode M. Algebraic data type and process specification based on projection spaces. In: Recent trends in data type specifications. No. 332 in Lecture Notes in Computer Science. Springer; 1988. p. 23-43.

[28] Walters RFC. Sheaves and Cauchy-complete categories. Cahiers de Topologie et Geometrie Differentielle. 1981;22:283-286.

[29] Walters RFC. Sheaves on sites as Cauchy-complete categories. Journal of Pure and Applied Algebra. 1982;24:95-102.

[30] Wagner KR. Solving recursive domain equations with enriched categories. Carnegie Mellon University; July 1994. Technical Report CMU-CS-94-159.

[31] Wagner KR. Liminf convergence in $\Omega$-categories. Theoretical Computer Science. 1997;184:61-104. 
[32] Majster-Cederbaum ME, Baier C. Metric completion versus ideal completion. Theoretical Computer Science. 1996;170:145-171.

[33] Kelley JL. General Topology. Springer Verlag; 1975.

[34] Mac Lane S. Categories for the Working Mathematician. Springer-Verlag; 1998.

[35] Barendregt HP, Dekkers WJM, Statman R. Lambda Calculus with Types. Perspectives in Mathematical Logic. Cambridge University Press/Association for Symbolic Logic; 2013.

[36] Winskel G. The Formal Semantics of Programming Languages: An Introduction. Cambridge, MA, USA: MIT Press; 1993.

[37] Kozen D. Coinductive Proof Principles for Stochastic Processes. Logical Methods in Computer Science. 2007;3(4).

[38] Kozen D, Ruozzi N. Applications of Metric Coinduction. Logical Methods in Computer Science. 2009;5(3).

[39] Dijkstra EW. Proving the equality of infinite sequences; 1991. Circulated privately, http://www.cs.utexas.edu/users/EWD/ewd10xx/EWD1093.PDF. 